\title{
ON THE DYNAMIC BEHAVIOR AND STABILITY OF CONTROLLED CONNECTED RAYLEIGH BEAMS UNDER POINTWISE OUTPUT FEEDBACK*
}

\author{
BaO-Zhu GuO ${ }^{1,2}$, Jun-Min WAnG $^{3}$ and Cui-Lian Zhou ${ }^{4}$
}

\begin{abstract}
We study the dynamic behavior and stability of two connected Rayleigh beams that are subject to, in addition to two sensors and two actuators applied at the joint point, one of the actuators also specially distributed along the beams. We show that with the distributed control employed, there is a set of generalized eigenfunctions of the closed-loop system, which forms a Riesz basis with parenthesis for the state space. Then both the spectrum-determined growth condition and exponential stability are concluded for the system. Moreover, we show that the exponential stability is independent of the location of the joint. The range of the feedback gains that guarantee the system to be exponentially stable is identified.
\end{abstract}

Mathematics Subject Classification. 93C20, 93C25, 35J10, 47E05

Received April 12, 2006. Revised October 23, 2006 and March 16, 2007.

Published online January 18, 2008.

\section{INTRODUCTION}

Pointwise stabilization of flexible structures has been studied extensively in the context of infinite-dimensional systems control over the past two decades due to wide applications in space technology and robotics $[1,2,5-10,14,15,28-30]$. Two fundamental issues, namely exponential stability and Riesz basis property, are investigated in these studies. We recall that Riesz basis property holds for a system if there exists a sequence of generalized eigenfunctions of the system, which forms a Riesz basis for the state space. The Riesz basis property is useful to deal with one dimensional vibrating systems that not only does it lead to results on stabilization, it also offers a deep insight into the dynamics of the system in terms of eigenfrequencies. Once the Riesz basis property is established, the exponential stability can be concluded directly and the growth rate can be determined in terms of the spectral abscissa. And one can often easily obtain the spectrum-determined growth condition (the earlier works for one-dimensional damping wave equation can be found in [11]); note that the latter does not hold for any partial differential equation systems [25] and its verification is known to be generally difficult.

\footnotetext{
Keywords and phrases. Rayleigh beam, collocated control, spectral analysis, exponential stability.

* Supported by the National Natural Science Foundation of China and the National Research Foundation of South Africa.

1 Academy of Mathematics and Systems Science, Academia Sinica, Beijing 100080, P.R. China; bzguo@iss.ac.cn

2 School of Computational and Applied Mathematics, University of the Witwatersrand, Wits 2050, Johannesburg, South Africa.

3 Corresponding author: Department of Mathematics, Beijing Institute of Technology, Beijing 100081, P.R. China; wangjc@graduate.hku.hk

4 Department of Mathematics, Beijing Institute of Technology, Beijing 100081, P.R. China.
} 
In this paper we are concerned with the following controlled two connected Rayleigh beams proposed in Weiss and Curtain [28]:

$$
\left\{\begin{array}{l}
y_{t t}(x, t)-\alpha y_{x x t}(x, t)+y_{x x x x}(x, t)=-u_{0}(t) \frac{\mathrm{d}}{\mathrm{d} x} \delta_{\xi}-\dot{u}_{1}(t)\left[\alpha \delta_{\xi}+b(x)\right], x \in(0,1) \\
y(0, t)=y_{x x}(0, t)=y(1, t)=y_{x x}(1, t)=0
\end{array}\right.
$$

where

$$
b(x):=\left\{\begin{array}{l}
(1-\xi) x, 0 \leq x \leq \xi \\
\xi(1-x), \xi<x \leq 1
\end{array}\right.
$$

$\delta_{\xi}, \frac{\mathrm{d} \delta_{\xi}}{\mathrm{d} x}$ are Dirac delta functions and the derivative at $x=\xi$ in the sense of distribution, $y(x, t)$ represents the transverse displacement of the beam at position $x \in[0,1]$ and time $t \geq 0, \alpha>0$ is a constant (which is proportional to the moment of inertia of the cross section of the beam), and $u_{0}, u_{1}$ are control inputs. Weiss and Curtain [28] designed the following feedback controls (with $k=k_{0}$ ):

$$
u_{0}(t)=-k_{0} y_{x t}(\xi, t), u_{1}(t)=-k\left[(1-\gamma) y_{x x}\left(\xi^{-}, t\right)+\gamma y_{x x}\left(\xi^{+}, t\right)\right]
$$

where $\gamma$ and the feedback gains $k, k_{0}$ are positive constants.

It is known (see e.g., [2] and also [6-8,24] for connected beams) that the system (1.1) is equivalent to the following Rayleigh beam equation:

$$
\left\{\begin{array}{l}
y_{t t}(x, t)-\alpha y_{x x t t}(x, t)+y_{x x x x}(x, t)=-\dot{u}_{1}(t) b(x), x \in(0,1), x \neq \xi \\
y(0, t)=y_{x x}(0, t)=y(1, t)=y_{x x}(1, t)=0 \\
y\left(\xi^{-}, t\right)=y\left(\xi^{+}, t\right), y_{x}\left(\xi^{-}, t\right)=y_{x}\left(\xi^{+}, t\right) \\
y_{x x}\left(\xi^{-}, t\right)-y_{x x}\left(\xi^{+}, t\right)=u_{0}(t) \\
y_{x x x}\left(\xi^{-}, t\right)-y_{x x x}\left(\xi^{+}, t\right)=\alpha \dot{u}_{1}(t) .
\end{array}\right.
$$

It is easy to see that there are two actuators involved in the system (1.4). One is imposed at the joint point and another is also imposed at the joint point but specially distributed along the entire beam at the same time. Due to the increasing application of smart materials, the distributed measurement and distributed control becomes feasible [20,21].

Using the newly developed result on collocated static output feedback in [13], Weiss and Curtain [28] showed that this distributed control $\dot{u}_{1}(t) b(x)$ in (1.1) together with the pointwise controls at the joint does exponentially stabilize the system (1.1), (1.3) and that the control (1.3) is robust to the position of the joint point. They obtained this result under the condition that the static output feedback gains lie in a suitable finite range. Precisely, the system (1.1) under (1.3) is exponentially stable if $k=k_{0} \in(0,2 /|\gamma-\xi|)$. It is not clear what would happen when the feedback gains are out of this range for both well-posedness and stability.

Let us look at the energy of the system (1.1) that is given by

$$
E(t)=\frac{1}{2} \int_{0}^{1}\left\{y_{x x}^{2}(x, t)+\left[y_{t}(x, t)+u_{1}(t) b(x)\right]^{2}+\alpha\left[y_{x t}(x, t)+u_{1}(t) b^{\prime}(x)\right]^{2}\right\} \mathrm{d} x .
$$


Formally, differentiate $E(t)$ with respect to time $t$ along the trajectory of (1.4), to give

$$
\begin{aligned}
\dot{E}(t)= & \int_{0}^{1}\left\{y_{x x} y_{x x t}+\left[\alpha y_{x x t t}-y_{x x x x}\right]\left[y_{t}+u_{1} b\right]+\alpha\left[y_{x t t}+\dot{u}_{1} b^{\prime}\right]\left[y_{x t}+u_{1} b^{\prime}\right]\right\} \mathrm{d} x \\
= & \int_{0}^{1}\left\{y_{x x} y_{x x t}+\alpha y_{x x t t} y_{t}+\alpha y_{x t t} y_{x t}-y_{x x x x} y_{t}\right. \\
& \left.+\alpha u_{1} y_{x t t} b^{\prime}+\alpha y_{x t} \dot{u}_{1} b^{\prime}+\alpha u_{1} \dot{u}_{1} b^{\prime 2}+\alpha u_{1} y_{x x t t} b-u_{1} y_{x x x x} b\right\} \mathrm{d} x \\
= & -\alpha \dot{u}_{1} y_{t}(\xi, t)-k_{0} y_{x t}^{2}(\xi, t) \\
& +\int_{0}^{1}\left\{\alpha u_{1} y_{x t t} b^{\prime}+\alpha y_{x t} \dot{u}_{1} b^{\prime}+\alpha u_{1} \dot{u}_{1} b^{\prime 2}+\alpha u_{1} y_{x x t t} b-u_{1} y_{x x x x} b\right\} \mathrm{d} x \\
= & -\alpha \dot{u}_{1} y_{t}(\xi, t)-k_{0} y_{x t}^{2}(\xi, t) \\
& +\alpha u_{1} y_{t t}(\xi, t)+\alpha \dot{u}_{1} y_{t}(\xi, t)+\alpha \xi(1-\xi) u_{1} \dot{u}_{1}-\alpha u_{1} y_{t t}(\xi, t)-\alpha \xi(1-\xi) u_{1} \dot{u}_{1} \\
& +u_{1} y_{x x}\left(\xi^{-}, t\right)+k_{0} \xi u_{1} y_{x t}(\xi, t) \\
= & -k_{0} y_{x t}^{2}(\xi, t)+u_{1} y_{x x}\left(\xi^{-}, t\right)+k_{0} \xi u_{1} y_{x t}(\xi, t) \\
= & -k_{0}\left(1+k_{0} k \gamma \xi\right) y_{x t}^{2}(\xi, t)-k y_{x x}^{2}\left(\xi^{-}, t\right)-k k_{0}(\gamma+\xi) y_{x x}\left(\xi^{-}, t\right) y_{x t}(\xi, t) \\
\leq & -k_{0}\left(1+k k_{0} \xi \gamma-\frac{k(\xi+\gamma)}{2 \delta}\right) y_{x t}^{2}(\xi, t)-k\left(1-\frac{\delta k_{0}(\xi+\gamma)}{2}\right) y_{x x}^{2}\left(\xi^{-}, t\right)
\end{aligned}
$$

for any $\delta>0$. It is seen that $\dot{E}(t) \leq 0$ provided that

$$
\frac{k(\xi+\gamma)}{2 \delta} \leq 1+k k_{0} \xi \gamma, \quad \frac{\delta k_{0}(\xi+\gamma)}{2} \leq 1 .
$$

The dissipativity of the closed-loop system (1.1) and (1.3) under the condition: $k k_{0}(\xi-\gamma)^{2} \leq 4$ will be proven rigorously as Lemma 3.2 in Section 4.

The main objective of this paper is to establish the Riesz basis property for the closed-loop Rayleigh beam described (1.1) under the feedback (1.3). We then conclude for the system (a) the spectrum-determined growth condition, (b) the exponential stability, and (c) the robustness to the position of joint point. To answer the question about the range of feedback gains, we show that if $k=k_{0}=2 /|\gamma-\xi|$, then there always exists a joint point $\xi \in(0,1)$ such that the system (1.1) under (1.3) is not exponentially stable. This sets up a constraint on the feedback gains. The earlier similar result for one dimensional nonhomogeneous wave equation can be found in [12].

Using results on the sharp trace regularity, Ammari and Tucsnak [1] proved the exponential stability for an Euler-Bernoulli beam under some conditions. Guo and Chan [15] established the Riesz basis property for Euler-Bernoulli beams with various boundary conditions. Xu and Yung [30] considered a Timoshenko beam with pointwise feedback control. It is pointed out that only one point control is implemented in these studies, and that the exponential stability is shown to be not robust to the location of the joint [2]; in other words, the stability results are dependent on the exact location of the joint point.

In order to achieve robust control, Ammari, Liu and Tucsnak [2] proposed to place two sensors and to use two actuators at the joint point $(x=\xi)$ in their study of stabilization of connected Rayleigh beams (Euler-Bernoulli beam as well):

$$
\left\{\begin{array}{l}
y_{t t}(x, t)-y_{x x t t}(x, t)+y_{x x x x}(x, t)+y_{t}(\xi, t) \delta_{\xi}-y_{x t}(x, t) \frac{\mathrm{d} \delta_{\xi}}{\mathrm{d} x}=0,0<\xi<\pi, x \in(0, \pi), \\
y(0, t)=y_{x x}(0, t)=y(\pi, t)=y_{x x}(\pi, t)=0 .
\end{array}\right.
$$


By the energy multiplier technique and frequency domain method, they showed that the exponential stability holds for the Euler-Bernoulli beam and is robust to the position of the joint. Unfortunately, the exponential stability for the Rayleigh beam holds only when the joint point belongs to a special subset of the beam occupation that is either countable or dense. As a result, the stability of the closed-loop system under output feedback control for two connected Rayleigh beams with two sensors and two actuators at one joint point is not robust to the location of the joint. In order to solve this problem, Weiss and Curtain [28] introduced an additional specially distributed control in (1.1).

We proceed as follows. In Section 2, the system is formulated into an evolution equation in the energy state space. The main results are stated in Section 3. Finally, in Section 4, we give the proofs of the main results.

\section{Problem formulation}

Motivated by the energy function (1.5) of the system (1.4), we define the state Hilbert space $\mathcal{H}$ for the system (1.1) as follows:

$$
\mathcal{H}:=\left(H^{2}(0,1) \cap H_{0}^{1}(0,1)\right) \times H_{0}^{1}(0,1),
$$

which is equipped with the inner product induced norm:

$$
\|(f, g)\|^{2}=\int_{0}^{1}\left[\left|f^{\prime \prime}(x)\right|^{2}+|g(x)|^{2}+\alpha\left|g^{\prime}(x)\right|^{2}\right] \mathrm{d} x, \forall(f, g) \in \mathcal{H} .
$$

Now, define the operator $\mathcal{R}: L^{2}(0,1) \rightarrow H^{2}(0,1) \cap H_{0}^{1}(0,1):$

$$
\mathcal{R}:=\left(I-\alpha \frac{\mathrm{d}^{2}}{\mathrm{~d} x^{2}}\right)^{-1}
$$

It is well-known that $\mathcal{R}$ is an isomorphism from $L^{2}(0,1)$ to $H^{2}(0,1) \cap H_{0}^{1}(0,1)[13,27,28]$ and

$$
\left\{\begin{array}{l}
\mathcal{R} f=c \sinh \frac{x}{\sqrt{\alpha}}-\frac{1}{\sqrt{\alpha}} \int_{0}^{x} \sinh \frac{x-s}{\sqrt{\alpha}} f(s) \mathrm{d} s \\
c=\left(\sqrt{\alpha} \sinh \frac{1}{\sqrt{\alpha}}\right)^{-1} \int_{0}^{1} \sinh \frac{1-s}{\sqrt{\alpha}} f(s) \mathrm{d} s, \forall f \in L^{2}(0,1) .
\end{array}\right.
$$

A simple computation shows that

$$
\left\{\begin{array}{l}
\left(\mathcal{R} \frac{\mathrm{d}^{4}}{\mathrm{~d} x^{4}}\right) f(x)=d \sinh \frac{x}{\sqrt{\alpha}}-\frac{1}{\alpha} f^{\prime \prime}(x)-\frac{1}{\alpha \sqrt{\alpha}} \int_{0}^{x} \sinh \frac{x-s}{\sqrt{\alpha}} f^{\prime \prime}(s) \mathrm{d} s \\
d=\left(\alpha \sinh \frac{1}{\sqrt{\alpha}}\right)^{-1} \int_{0}^{1} \cosh \frac{1-s}{\sqrt{\alpha}} f^{\prime \prime \prime}(s) \mathrm{d} s
\end{array}\right.
$$

Next, apply $\mathcal{R}$ to both sides of (1.1) to obtain (see (5.3) of [28]):

$$
\left\{\begin{array}{l}
y_{t t}(x, t)+\left(\mathcal{R} \frac{\mathrm{d}^{4}}{\mathrm{~d} x^{4}}\right) y(x, t)=-u_{0}(t) \mathcal{R}\left(\frac{\mathrm{d}}{\mathrm{d} x} \delta_{\xi}\right)-\dot{u}_{1}(t) b(x), \\
y(0, t)=y_{x x}(0, t)=y(1, t)=y_{x x}(1, t)=0
\end{array}\right.
$$


where $\mathcal{R}\left(\frac{\mathrm{d}}{\mathrm{d} x} \delta_{\xi}\right)$, by extending $\mathcal{R}$ as $\mathcal{R} \in \mathcal{L}\left(\left(H^{2}(0,1) \cap H_{0}^{1}(0,1)\right)^{\prime}, L^{2}(0,1)\right)$ (see e.g. [13], p. 294), is computed to be (see e.g., (3.15) of [2]):

$$
\mathcal{R}\left(\frac{\mathrm{d}}{\mathrm{d} x} \delta_{\xi}\right)=\left\{\begin{array}{l}
\left(\alpha \sinh \frac{1}{\sqrt{\alpha}}\right)^{-1} \cosh \frac{1-\xi}{\sqrt{\alpha}} \sinh \frac{x}{\sqrt{\alpha}}, \quad x \in(0, \xi), \\
\left(\alpha \sinh \frac{1}{\sqrt{\alpha}}\right)^{-1} \cosh \frac{1-\xi}{\sqrt{\alpha}} \sinh \frac{x}{\sqrt{\alpha}}-\frac{1}{\alpha} \cosh \frac{x-\xi}{\sqrt{\alpha}}, x \in(\xi, 1) .
\end{array}\right.
$$

Thus, (2.5) can be rewritten as

$$
\left\{\begin{array}{l}
\frac{\mathrm{d}}{\mathrm{d} t}\left[y_{t}(x, t)+u_{1}(t) b(x)\right]+\left(\mathcal{R} \frac{\mathrm{d}^{4}}{\mathrm{~d} x^{4}}\right) y(x, t)=-u_{0}(t) \mathcal{R}\left(\frac{\mathrm{d}}{\mathrm{d} x} \delta_{\xi}\right) \\
y(0, t)=y_{x x}(0, t)=y(1, t)=y_{x x}(1, t)=0
\end{array}\right.
$$

or equivalently

$$
\left\{\begin{array}{c}
\frac{\mathrm{d}}{\mathrm{d} t}\left[\begin{array}{c}
y(x, t) \\
y_{t}(x, t)+u_{1}(t) b(x)
\end{array}\right]+\left[\left(\begin{array}{cc}
0 & -I \\
\left(\mathcal{R} \frac{\mathrm{d}^{4}}{\mathrm{~d} x^{4}}\right) & 0
\end{array}\right]\left[\begin{array}{c}
y(x, t) \\
y_{t}(x, t)+u_{1}(t) b(x)
\end{array}\right]\right. \\
+\left[\begin{array}{cc}
0 & b(x) \\
\mathcal{R}\left(\frac{\mathrm{d}}{\mathrm{d} x} \delta_{\xi}\right) & 0
\end{array}\right]\left[\begin{array}{l}
u_{0}(t) \\
u_{1}(t)
\end{array}\right]=0 \\
y(0, t)=y_{x x}(0, t)=y(1, t)=y_{x x}(1, t)=0 .
\end{array}\right.
$$

Substitute (1.3) into (2.8), we get naturally the system operator $\mathcal{A}: D(\mathcal{A})(\subset \mathcal{H}) \rightarrow \mathcal{H}$ of $(1.1),(1.3)$ as

$$
\begin{aligned}
& D(\mathcal{A})=\left\{(f, g) \mid \mathcal{A}(f, g) \in \mathcal{H}, f \in H^{3}(0, \xi) \cup H^{3}(\xi, 1), f^{\prime \prime}(0)=f^{\prime \prime}(1)=0\right\}, \\
& \mathcal{A}\left[\begin{array}{c}
f \\
g
\end{array}\right]=\left\{\begin{array}{l}
{\left[\begin{array}{l}
g+b \tilde{f}(\xi) \\
-\left(\mathcal{R} \frac{\mathrm{d}^{4}}{\mathrm{~d} x^{4}}\right) f+k_{0} \mathcal{R}\left(\frac{\mathrm{d}}{\mathrm{d} x} \delta_{\xi}\right)\left[g^{\prime}\left(\xi^{-}\right)+(1-\xi) \widetilde{f}(\xi)\right]
\end{array}\right], \quad 0 \leq x<\xi,} \\
{\left[\begin{array}{l}
g+b \widetilde{f}(\xi) \\
-\left(\mathcal{R} \frac{\mathrm{d}^{4}}{\mathrm{~d} x^{4}}\right) f+k_{0} \mathcal{R}\left(\frac{\mathrm{d}}{\mathrm{d} x} \delta_{\xi}\right)\left[g^{\prime}\left(\xi^{+}\right)-\xi \widetilde{f}(\xi)\right]
\end{array}\right], \quad \xi \leq x \leq 1,} \\
\tilde{f}(\xi):=k\left[(1-\gamma) f^{\prime \prime}\left(\xi^{-}\right)+\gamma f^{\prime \prime}\left(\xi^{+}\right)\right] .
\end{array}\right.
\end{aligned}
$$

The above expression can be further simplified. Actually, by $\mathcal{A}(f, g) \in \mathcal{H}$, one has

$$
g^{\prime}\left(\xi^{-}\right)+k(1-\xi)\left[(1-\gamma) f^{\prime \prime}\left(\xi^{-}\right)+\gamma f^{\prime \prime}\left(\xi^{+}\right)\right]=g^{\prime}\left(\xi^{+}\right)-k \xi\left[(1-\gamma) f^{\prime \prime}\left(\xi^{-}\right)+\gamma f^{\prime \prime}\left(\xi^{+}\right)\right]
$$

or

$$
g^{\prime}\left(\xi^{+}\right)-g^{\prime}\left(\xi^{-}\right)=k\left[(1-\gamma) f^{\prime \prime}\left(\xi^{-}\right)+\gamma f^{\prime \prime}\left(\xi^{+}\right)\right]
$$

and

$$
\begin{aligned}
& \frac{1}{\alpha} f^{\prime \prime}\left(\xi^{-}\right)+k_{0} \mathcal{R}\left(\frac{\mathrm{d}}{\mathrm{d} x} \delta_{\xi}\right)\left(\xi^{-}\right)\left[g^{\prime}\left(\xi^{-}\right)+k(1-\xi)\left[(1-\gamma) f^{\prime \prime}\left(\xi^{-}\right)+\gamma f^{\prime \prime}\left(\xi^{+}\right)\right]\right] \\
& =\frac{1}{\alpha} f^{\prime \prime}\left(\xi^{+}\right)+k_{0} \mathcal{R}\left(\frac{\mathrm{d}}{\mathrm{d} x} \delta_{\xi}\right)\left(\xi^{+}\right)\left[g^{\prime}\left(\xi^{+}\right)-k \xi\left[(1-\gamma) f^{\prime \prime}\left(\xi^{-}\right)+\gamma f^{\prime \prime}\left(\xi^{+}\right)\right]\right],
\end{aligned}
$$


where

$$
\mathcal{R}\left(\frac{\mathrm{d}}{\mathrm{d} x} \delta_{\xi}\right)\left(\xi^{+}\right)=L-\frac{1}{\alpha} \text { with } L=\mathcal{R}\left(\frac{\mathrm{d}}{\mathrm{d} x} \delta_{\xi}\right)\left(\xi^{-}\right)=\left(\alpha \sinh \frac{1}{\sqrt{\alpha}}\right)^{-1} \cosh \frac{1-\xi}{\sqrt{\alpha}} \sinh \frac{\xi}{\sqrt{\alpha}} .
$$

Thus

Therefore

$$
\left\{\begin{array}{l}
g^{\prime}\left(\xi^{+}\right)=k \xi\left[(1-\gamma) f^{\prime \prime}\left(\xi^{-}\right)+\gamma f^{\prime \prime}\left(\xi^{+}\right)\right]-\frac{1}{k_{0}}\left[f^{\prime \prime}\left(\xi^{-}\right)-f^{\prime \prime}\left(\xi^{+}\right)\right] \\
g^{\prime}\left(\xi^{-}\right)=-k(1-\xi)\left[(1-\gamma) f^{\prime \prime}\left(\xi^{-}\right)+\gamma f^{\prime \prime}\left(\xi^{+}\right)\right]-\frac{1}{k_{0}}\left[f^{\prime \prime}\left(\xi^{-}\right)-f^{\prime \prime}\left(\xi^{+}\right)\right]
\end{array}\right.
$$

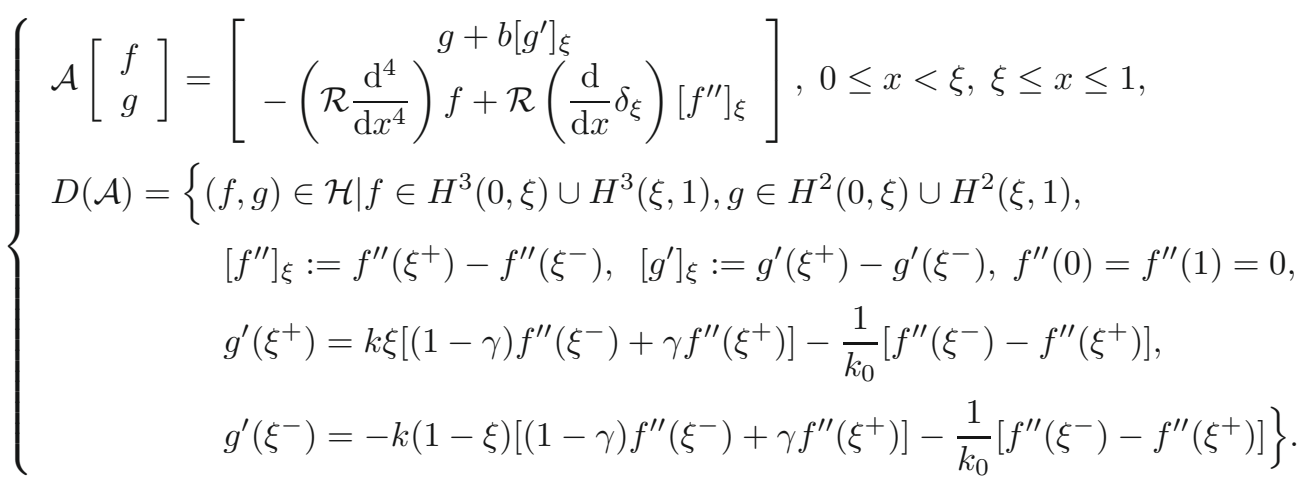

With the operator $\mathcal{A}$ at hand, the closed-loop system (1.1) under the feedback controls (1.3) can be formulated into the following abstract evolution equation in $\mathcal{H}$ :

$$
\left\{\begin{array}{l}
\dot{Y}(t)=\mathcal{A} Y(t) \\
Y(0)=Y_{0}
\end{array}\right.
$$

where $Y(t):=\left(y(\cdot, t), y_{t}(\cdot, t)+u_{1}(t) b(\cdot)\right)$ and $Y_{0}$ is the initial datum.

\section{MAin RESUlts}

In this section, we state the main results as well as some main preliminary lemmas to be used for the proofs of the main results of this paper. All these proofs are given in Section 4.

To begin with, let us recall that for an (unbounded) operator $\mathbf{A}$ defined in $\mathcal{H}, W=(f, g) \in D(\mathbf{A})$ is said to be a generalized eigenvector of $\mathbf{A}$ associated with an eigenvalue $\lambda$ if there is an integer $\ell \geq 1$ such that $(\lambda-\mathbf{A})^{\ell} W=0$. The root subspace of $\mathbf{A}$ that is denoted by $\operatorname{Sp}(\mathbf{A})$, is the closed subspace of $\mathcal{H}$ spanned by all generalized eigenfunctions of $\mathbf{A}$. The root subspace is said to be complete in $\mathcal{H}$ if $\operatorname{Sp}(\mathbf{A})=\mathcal{H}$. The integer $m_{(a)}(\lambda)=\operatorname{dim}\left\{W \mid(\lambda-\mathbf{A})^{\ell} W=0\right.$ for some integer $\left.\ell\right\}$ is called the algebraic multiplicity of $\lambda$. $\lambda$ is said to be algebraically simple if $m_{(a)}(\lambda)=1$. It is well-known that each eigenvalue of a discrete operator (that is, there is a $\lambda \in \sigma(\mathbf{A})$, the spectrum set of $\mathbf{A}$, such that $(\lambda-\mathbf{A})^{-1}$ is compact on $\left.\mathcal{H}\right)$ must have finite algebraic multiplicity. The algebraic multiplicity can be represented through eigen-projection. Let $\Gamma$ be a circle and let $\lambda \in \sigma_{p}(\mathbf{A})$, the point spectrum set of $\mathbf{A}$, be the unique spectrum of $\mathbf{A}$ inside of $\Gamma$. Then the eigen-projection $\mathbb{P}_{\lambda}$ is defined as

$$
\mathbb{P}_{\lambda}=\frac{1}{2 \pi i} \int_{\Gamma}(s-\mathbf{A})^{-1} \mathrm{~d} s
$$

and $m_{(a)}(\lambda)=\operatorname{dim} \mathbb{P}_{\lambda} \mathcal{H}$. A nonzero $W \in D(\mathbf{A})$ is called an eigenvector of $\mathbf{A}$ corresponding to the eigenvalue $\lambda$ if $(\lambda-\mathbf{A}) W=0$. The number $m_{(g)}(\lambda)=\operatorname{dim}\{W \mid(\lambda-\mathbf{A}) W=0\}$ is called the geometric multiplicity of $\lambda$. $\lambda$ is said to be geometrically simple if $m_{(g)}(\lambda)=1$. 
Lemma 3.1. Let $\mathcal{A}$ be defined by (2.13). Then $\mathcal{A}^{-1}$ exists and is compact on $\mathcal{H}$ and hence $\mathcal{A}$ is a discrete operator in $\mathcal{H}$. Therefore, $\sigma(\mathcal{A})$ consists of isolated eigenvalues with finite algebraic multiplicities only.

Lemma 3.2. If $k$ and $k_{0}$ satisfy the following condition

$$
k k_{0}(\xi-\gamma)^{2} \leq 4
$$

then $\mathcal{A}$ is dissipative and hence $\mathcal{A}$ generates a $C_{0}$-semigroup of contractions on $\mathcal{H}$. If in addition

$$
k k_{0}(\xi-\gamma)^{2}<4
$$

then $\operatorname{Re}(\lambda)<0$ for any $\lambda \in \sigma(\mathcal{A})$.

Remark 3.3. When $k=k_{0},(3.2)$ is reduced to be

$$
k \in(0, \tilde{k}), \quad \tilde{k}=\frac{2}{|\gamma-\xi|} .
$$

This is just the condition in Theorem 1.1 of [28].

Now we formulate the eigenvalue problem for $\mathcal{A}$. Let $\lambda \in \sigma(\mathcal{A})$ and $(f, g)$ be its corresponding eigenfunction: $\mathcal{A}(f, g)=\lambda(f, g)$. Then

$$
g=\lambda f-k b\left[(1-\gamma) f^{\prime \prime}\left(\xi^{-}\right)+\gamma f^{\prime \prime}\left(\xi^{+}\right)\right],
$$

and $f$ solves the following eigenvalue problem:

$$
\left\{\begin{array}{l}
\lambda^{2} f(x)-\alpha \lambda^{2} f^{\prime \prime}(x)+f^{(4)}(x)=k \lambda b\left[(1-\gamma) f^{\prime \prime}\left(\xi^{-}\right)+\gamma f^{\prime \prime}\left(\xi^{+}\right)\right], x \in(0,1), x \neq \xi \\
f(0)=f^{\prime \prime}(0)=f(1)=f^{\prime \prime}(1)=0 \\
f\left(\xi^{-}\right)=f\left(\xi^{+}\right), f^{\prime}\left(\xi^{-}\right)=f^{\prime}\left(\xi^{+}\right) \\
f^{\prime \prime}\left(\xi^{-}\right)-f^{\prime \prime}\left(\xi^{+}\right)=-k_{0} \lambda f^{\prime}(\xi) \\
f^{\prime \prime \prime}\left(\xi^{-}\right)-f^{\prime \prime \prime}\left(\xi^{+}\right)=-\alpha k \lambda\left[(1-\gamma) f^{\prime \prime}\left(\xi^{-}\right)+\gamma f^{\prime \prime}\left(\xi^{+}\right)\right]
\end{array}\right.
$$

Differentiate (3.5) twice, to obtain

$$
\left\{\begin{array}{l}
f^{(6)}(x)-\alpha \lambda^{2} f^{(4)}(x)+\lambda^{2} f^{\prime \prime}(x)=0, x \in(0,1), x \neq \xi \\
f(0)=f^{\prime \prime}(0)=f^{(4)}(0)=f(1)=f^{\prime \prime}(1)=f^{(4)}(1)=0 \\
f\left(\xi^{-}\right)=f\left(\xi^{+}\right), f^{\prime}\left(\xi^{-}\right)=f^{\prime}\left(\xi^{+}\right) \\
f^{\prime \prime}\left(\xi^{-}\right)-f^{\prime \prime}\left(\xi^{+}\right)=-k_{0} \lambda f^{\prime}(\xi) \\
f^{\prime \prime \prime}\left(\xi^{-}\right)-f^{\prime \prime \prime}\left(\xi^{+}\right)=-\alpha k \lambda\left[(1-\gamma) f^{\prime \prime}\left(\xi^{-}\right)+\gamma f^{\prime \prime}\left(\xi^{+}\right)\right] \\
f^{(4)}\left(\xi^{-}\right)-f^{(4)}\left(\xi^{+}\right)=-k_{0} \alpha \lambda^{3} f^{\prime}(\xi) \\
f^{(5)}\left(\xi^{-}\right)-f^{(5)}\left(\xi^{+}\right)=\left(k \lambda-k \alpha^{2} \lambda^{3}\right)\left[(1-\gamma) f^{\prime \prime}\left(\xi^{-}\right)+\gamma f^{\prime \prime}\left(\xi^{+}\right)\right]
\end{array}\right.
$$

Suppose $\lambda^{2} \neq 4 / \alpha^{2}$ and $\lambda \neq 0$. Let

$$
\tau_{1}(\lambda)=\sqrt{\frac{\alpha \lambda^{2}+\sqrt{\alpha^{2} \lambda^{4}-4 \lambda^{2}}}{2}}, \tau_{2}(\lambda)=\sqrt{\frac{\alpha \lambda^{2}-\sqrt{\alpha^{2} \lambda^{4}-4 \lambda^{2}}}{2}} .
$$

Then

$$
\left\{1, x, \sinh \tau_{1} x, \cosh \tau_{1} x, \sinh \tau_{2} x, \cosh \tau_{2} x\right\}
$$

is a set of fundamental solutions for the equation $f^{(6)}(x)-\alpha \lambda^{2} f^{(4)}(x)+\lambda^{2} f^{\prime \prime}(x)=0$. 
Theorem 3.4. There is a characteristic determinant $\operatorname{det}(\Delta(\lambda))$ for the eigenvalue problem (3.6) (that is to say, if $\lambda^{2} \neq 4 / \alpha^{2}, \lambda \neq 0$, then $\lambda \in \sigma(\mathcal{A})$ if and only if $\operatorname{det}(\Delta(\lambda))=0$ ) such that the following asymptotic expansion holds:

$$
\operatorname{det}(\Delta(\lambda))=-\lambda^{4} \tau_{1}^{4} \sinh \frac{1}{\sqrt{\alpha}}\left[\Delta_{1}(\lambda)+\mathcal{O}\left(\lambda^{-1}\right)\right] \text { as }|\lambda| \rightarrow \infty,
$$

where $\tau_{1}$ is given in (3.7), and

$$
\left\{\begin{aligned}
\Delta_{1}(\lambda)= & K_{1} \sinh (\sqrt{\alpha} \lambda)+K_{2} \cosh (\sqrt{\alpha} \lambda) \\
& +K_{3} \cosh (\sqrt{\alpha} \lambda(1-2 \xi))+K_{4} \sinh (\sqrt{\alpha} \lambda(1-2 \xi)), \\
K_{1}=1 & +\frac{k_{0} k \xi \gamma}{2}+\frac{k(1-\gamma) k_{0}(1-\xi)}{2}, K_{2}=\frac{\sqrt{\alpha} k}{2}+\frac{k_{0}}{2 \sqrt{\alpha}} \\
K_{3}= & \frac{k_{0}}{2 \sqrt{\alpha}}-\frac{\sqrt{\alpha} k}{2}, K_{4}=\frac{k_{0} k \xi \gamma}{2}-\frac{k(1-\gamma) k_{0}(1-\xi)}{2} .
\end{aligned}\right.
$$

Corollary 3.5. If $K_{1} \neq \pm K_{2}$, then the zeros of $\operatorname{det}(\Delta(\lambda))$ are located in a vertical strip parallel to the imaginary axis in the complex plane. In other words, there is a positive constant $C_{0}$ such that

$$
|\operatorname{Re}(\lambda)| \leq C_{0} \text { for any } \lambda \text { satisfying } \operatorname{det}(\Delta(\lambda))=0 .
$$

Theorem 3.6. Suppose condition (3.1) is fulfilled. Let $\mathcal{A}$ be defined by (2.13) and $K_{1}, K_{2}$ be given in (3.10). If $K_{1} \neq K_{2}$, then the root subspace of $\mathcal{A}$ is complete in $\mathcal{H}: \operatorname{Sp}(\mathcal{A})=\mathcal{H}$.

In what follows, we denote by $\mathcal{J}$ some set of integers, which may be different in different cases although they are denoted with the same symbol.

Recall that the sequence $\left\{W_{i}\right\}_{i \in \mathcal{J}}$ is called a basis for $\mathcal{H}$ if to each element $W \in \mathcal{H}$ corresponds a unique sequence of scalars $\left\{c_{i}\right\}$ such that the series

$$
W=\sum_{i \in \mathcal{J}} c_{i} W_{i}
$$

is convergent with respect to the norm of $\mathcal{H} .\left\{W_{i}\right\}_{i \in \mathcal{J}}$ is called a Riesz basis for $\mathcal{H}$ if

(a) $\operatorname{span}\left\{W_{i}\right\}=\mathcal{H}$;

(b) there exist some positive constants $m_{1}$ and $m_{2}$ such that for any numbers $c_{i}, i \in \mathcal{I}$, where $\mathcal{I}$ is any finite subset of $\mathcal{J}$, it has

$$
m_{1} \sum_{i \in \mathcal{I}}\left|c_{i}\right|^{2} \leq\left\|\sum_{i \in \mathcal{I}} c_{i} W_{i}\right\|^{2} \leq m_{2} \sum_{i \in \mathcal{I}}\left|c_{i}\right|^{2} .
$$

A basis $\left\{W_{i}\right\}_{i \in \mathcal{J}}$ for $\mathcal{H}$ is called a Riesz basis with parentheses [26] if (3.11) converges in $\mathcal{H}$ after putting some of its terms in parentheses the arrangement of which does not depend on $W$. We refer to [31] for more details on Riesz basis.

The following Theorem 3.7 is the main result of this paper.

Theorem 3.7. Suppose condition (3.1) is fulfilled. Let $K_{1}, K_{2}$ be given in (3.10). If $K_{1} \neq K_{2}$, then the following assertions hold.

(a) There exists a $\varepsilon>0$ such that

$$
\sigma(\mathcal{A})=\bigcup_{p \in \mathcal{J}}\left\{\lambda_{i}^{p}\right\}_{i=1}^{N^{p}}
$$

where $\lambda_{i}^{p} \neq \lambda_{j}^{p}$ whenever $i \neq j, N^{p}$ are integers satisfying $\sup _{p} N^{p}<\infty$, and

$$
\inf _{p \neq q, p, q \in \mathcal{J}}\left|\lambda_{i}^{p}-\lambda_{j}^{q}\right| \geq \varepsilon, \quad \forall 1 \leq i \leq N^{p}, 1 \leq j \leq N^{q} .
$$


(b) There is a set of generalized eigenfunctions of $\mathcal{A}$, which forms a Riesz basis with parentheses for $\mathcal{H}$. More precisely,

$$
W=\sum_{p \in \mathcal{J}} \sum_{i=1}^{N^{p}} \mathbb{P}_{\lambda_{i}^{p}} W, \forall W \in \mathcal{H}
$$

and there are constants $M_{1}, M_{2}>0$ such that

$$
M_{1} \sum_{p \in \mathcal{J}}\left\|\sum_{i=1}^{N^{p}} \mathbb{P}_{\lambda_{i}^{p}} W\right\|^{2} \leq\|W\|^{2} \leq M_{2} \sum_{p \in \mathcal{J}}\left\|\sum_{i=1}^{N^{p}} \mathbb{P}_{\lambda_{i}^{p}} W\right\|^{2}, \forall W \in \mathcal{H} .
$$

(c) The spectrum-determined growth condition holds true $[22]: S(\mathcal{A})=\omega(\mathcal{A})$, where

$$
S(\mathcal{A}):=\sup _{\lambda \in \sigma(\mathcal{A})} \operatorname{Re} \lambda
$$

is the spectral bound of $\mathcal{A}$, and

$$
\omega(\mathcal{A}):=\inf \left\{\omega \mid \exists M>0 \text { such that }\left\|\mathrm{e}^{\mathcal{A} t}\right\| \leq M \mathrm{e}^{\omega t}\right\}
$$

is the growth order of $\mathrm{e}^{\mathcal{A} t}$.

Theorem 3.8. Let $K_{1}, K_{2}$ be given in (3.10). If $K_{1} \neq K_{2}$, then under the condition (3.2), the imaginary axis is not the asymptote of eigenvalues of $\mathcal{A}$. Therefore, the system (2.14) is exponentially stable in the sense of

$$
\|Y(t)\| \leq M \mathrm{e}^{-\omega t}\|Y(0)\|
$$

for some positive numbers $M, \omega$.

Remark 3.9. For the completeness of root subspace and Riesz basis generation, we always assume that $K_{1} \neq K_{2}$, where $K_{1}, K_{2}$ are given in (3.10). This is standard for wave equation with same order feedback [16] since otherwise, $\sigma(\mathcal{A})$ may be empty (see (3.9) and (4.17)). For instance, when $K_{1}=K_{2}, \xi=1 / 2, \Delta_{1}(\lambda)$ given in (3.10) becomes

Thus when $K_{3}=0,(3.9)$ becomes

$$
\Delta_{1}(\lambda)=K_{3}+K_{1} \mathrm{e}^{\sqrt{\alpha} \lambda}
$$

$$
\operatorname{det}(\Delta(\lambda))=-\lambda^{4} \tau_{1}^{4} \sinh \frac{1}{\sqrt{\alpha}}\left[K_{1} \mathrm{e}^{\sqrt{\alpha} \lambda}+\mathcal{O}\left(\lambda^{-1}\right)\right] \text { as }|\lambda| \rightarrow \infty .
$$

We could not get information about the distribution of spectrum of $\mathcal{A}$ although we do not know whether $\sigma(\mathcal{A})$ is empty or not.

Theorem 3.8 is the main result of [28]. Finally, we answer the question proposed by Curtain and Weiss [28], which is a special case of $k k_{0}(\xi-\gamma)^{2}=4$ in (3.1) with $k=k_{0}$. This sets up a constraint on the feedback gains.

Theorem 3.10. Suppose $k k_{0}(\xi-\gamma)^{2}=4$ and $\gamma=2-\xi$. Then there is a $\xi \in(0,1)$ such that the system (2.14) is not exponentially stable.

\section{Proofs of the MAin Results}

Consider the following Volterra integral equation

$$
F_{0}(x)+\frac{1}{\sqrt{\alpha}} \int_{0}^{x} \sinh \frac{x-s}{\sqrt{\alpha}} F_{0}(s) \mathrm{d} s=G(x), \quad x \in[0,1] .
$$


It is well-known that for any $G \in L^{2}(0,1)$, there exists a unique continuous solution $F_{0}$ to the equation (4.1), which is denoted by

$$
F_{0}(x)=\left[(I+K)^{-1} G\right](x), \quad x \in[0,1],
$$

where $K$ is a compact operator on $L^{2}(0,1)$ defined in an obvious way from (4.1).

Lemma 4.1. Let $F_{0}, G$ be defined in (4.2). Then

$$
\left\{\begin{array}{l}
(I+K)^{-1} \sinh \frac{x}{\sqrt{\alpha}}=\frac{1}{\sqrt{\alpha}} x \\
F_{0} \in H^{1}(0,1) \text { whenever } G \in H^{1}(0,1) .
\end{array}\right.
$$

Proof. A straightforward computation gives the required result. We omit the details here.

Proof of Lemma 3.1. For any given $(\phi, \psi) \in \mathcal{H}, \mathcal{A}(f, g)=(\phi, \psi)$ means that

$$
\left\{\begin{array}{l}
g+k b\left[(1-\gamma) f^{\prime \prime}\left(\xi^{-}\right)+\gamma f^{\prime \prime}\left(\xi^{+}\right)\right]=\phi \\
-\left(\mathcal{R} \frac{\mathrm{d}^{4}}{\mathrm{~d} x^{4}}\right) f-\mathcal{R}\left(\frac{\mathrm{d}}{\mathrm{d} x} \delta_{\xi}\right)\left[f^{\prime \prime}\left(\xi^{-}\right)-f^{\prime \prime}\left(\xi^{+}\right)\right]=\psi, x \in(0,1), x \neq \xi
\end{array}\right.
$$

Since $(f, g) \in D(\mathcal{A})$, it follows from the first equation of (4.4) that

$$
f^{\prime \prime}\left(\xi^{-}\right)-f^{\prime \prime}\left(\xi^{+}\right)=-k_{0} \phi^{\prime}(\xi)
$$

by which the second equation of (4.4) becomes

$$
\left(\mathcal{R} \frac{\mathrm{d}^{4}}{\mathrm{~d} x^{4}}\right) f=k_{0} \phi^{\prime}(\xi) \mathcal{R}\left(\frac{\mathrm{d}}{\mathrm{d} x} \delta_{\xi}\right)-\psi, x \in(0,1), x \neq \xi
$$

By (2.4), the above can be written as

$$
d \alpha \sinh \frac{x}{\sqrt{\alpha}}-f^{\prime \prime}(x)-\frac{1}{\sqrt{\alpha}} \int_{0}^{x} \sinh \frac{x-s}{\sqrt{\alpha}} f^{\prime \prime}(s) \mathrm{d} s=k_{0} \alpha \phi^{\prime}(\xi) \mathcal{R}\left(\frac{\mathrm{d}}{\mathrm{d} x} \delta_{\xi}\right)-\alpha \psi
$$

for any $x \in[0,1], x \neq \xi$. Since $\psi(0)=0,(4.5)$ together with (2.6) gives $f^{\prime \prime}(0)=0$. By (4.1), (4.2) and Lemma 4.1, it has

$$
\begin{aligned}
f^{\prime \prime}(x) & =-\alpha(I+K)^{-1}\left[k_{0} \phi^{\prime}(\xi) \mathcal{R}\left(\frac{\mathrm{d}}{\mathrm{d} x} \delta_{\xi}\right)-\psi\right](x)+d \alpha(I+K)^{-1} \sinh \frac{x}{\sqrt{\alpha}} \\
& =-\alpha(I+K)^{-1}\left[k_{0} \phi^{\prime}(\xi) \mathcal{R}\left(\frac{\mathrm{d}}{\mathrm{d} x} \delta_{\xi}\right)-\psi\right](x)+d \sqrt{\alpha} x, x \in(0,1), x \neq \xi
\end{aligned}
$$

Since $f^{\prime \prime}(1)=0$, the above implies that

$$
\left\{\begin{array}{l}
d=\sqrt{\alpha}(I+K)^{-1}\left[k_{0} \phi^{\prime}(\xi) \mathcal{R}\left(\frac{\mathrm{d}}{\mathrm{d} x} \delta_{\xi}\right)-\psi\right](1) \\
f^{\prime \prime}\left(\xi^{-}\right)=-\alpha(I+K)^{-1}\left[k_{0} \phi^{\prime}(\xi) \mathcal{R}\left(\frac{\mathrm{d}}{\mathrm{d} x} \delta_{\xi}\right)-\psi\right]\left(\xi^{-}\right)+d \sqrt{\alpha} \xi
\end{array}\right.
$$


Hence

$$
\left\{\begin{array}{l}
f(x)=\int_{1}^{x}(x-s) f^{\prime \prime}(s) \mathrm{d} s+(x-1) \int_{0}^{1} s f^{\prime \prime}(s) \mathrm{d} s \\
f^{\prime \prime}(x)=-\alpha(I+K)^{-1}\left[k_{0} \phi^{\prime}(\xi) \mathcal{R}\left(\frac{\mathrm{d}}{\mathrm{d} x} \delta_{\xi}\right)-\psi\right](x)+d \sqrt{\alpha} x, \quad x \in(0,1), x \neq \xi \\
g(x)=-k b(x) f^{\prime \prime}\left(\xi^{-}\right)-k k_{0} \gamma \phi^{\prime}(\xi) b(x)+\phi(x),
\end{array}\right.
$$

where $d, f^{\prime \prime}\left(\xi^{-}\right)$are given by (4.6). Now we claim that $(f, g) \in D(\mathcal{A})$. Indeed, due to the fact that

$$
\mathcal{R}\left(\frac{\mathrm{d}}{\mathrm{d} x} \delta_{\xi}\right) \in H_{0}^{1}(0,1) \backslash\{\xi\}, \quad \phi \in H^{2}(0,1) \cap H_{0}^{1}(0,1),
$$

it has

$$
f^{\prime \prime}(x) \in H_{0}^{1}(0,1) \backslash\{\xi\}, f \in H^{3}(0,1) \backslash\{\xi\}, g \in H^{2}(0,1) \backslash\{\xi\}
$$

Moreover,

$$
f(0)=f(1)=g(0)=g(1)=f^{\prime \prime}(0)=f^{\prime \prime}(1)=0,
$$

and $f^{\prime \prime}\left(\xi^{-}\right), f^{\prime \prime}\left(\xi^{+}\right), g^{\prime}\left(\xi^{-}\right)$and $g^{\prime}\left(\xi^{+}\right)$satisfy the conditions given in (2.13). Therefore, $(f, g) \in D(\mathcal{A})$ and $\mathcal{A}^{-1}(\phi, \psi)=(f, g)$ identified by (4.7). Finally, by the Sobolev embedding theorem, (4.7) implies that $\mathcal{A}^{-1}$ is compact, proving the required result.

Proof of Lemma 3.2. First, suppose condition (3.1) is fulfilled. Let $(f, g) \in D(\mathcal{A})$. Compute directly from (2.4) and (2.6) to obtain

$$
\left\langle\left(\mathcal{R} \frac{\mathrm{d}^{4}}{\mathrm{~d} x^{4}}\right) f, g\right\rangle_{H_{0}^{1}(0,1)}=-\int_{0}^{1} f^{\prime \prime \prime} \overline{g^{\prime}} \mathrm{d} x,\left\langle\left(\frac{\mathrm{d}}{\mathrm{d} x} \delta_{\xi}\right), g\right\rangle_{H_{0}^{1}(0,1)}=0
$$

Hence

$$
\begin{aligned}
& \left\langle\mathcal{A}\left[\begin{array}{l}
f \\
g
\end{array}\right],\left[\begin{array}{l}
f \\
g
\end{array}\right]\right\rangle=\left\langle\left[-\left(\mathcal{R} \frac{\mathrm{d}^{4}}{\mathrm{~d} x^{4}}\right) f+b\left[g^{\prime}\right]_{\xi}\left(\frac{\mathrm{d}}{\mathrm{d} x} \delta_{\xi}\right)\left[f^{\prime \prime}\right]_{\xi}\right],\left[\begin{array}{l}
f \\
g
\end{array}\right]\right\rangle \\
& =\int_{0}^{1}\left[g^{\prime \prime} \overline{f^{\prime \prime}}+f^{\prime \prime \prime} \overline{g^{\prime}}\right] \mathrm{d} x=\left.f^{\prime \prime} \overline{g^{\prime}}\right|_{0} ^{\xi}+\left.f^{\prime \prime} \overline{g^{\prime}}\right|_{\xi} ^{1}+\int_{0}^{1}\left[g^{\prime \prime} \overline{f^{\prime \prime}}-f^{\prime \prime} \overline{g^{\prime \prime}}\right] \mathrm{d} x \\
& =f^{\prime \prime}\left(\xi^{-}\right) \overline{g^{\prime}}\left(\xi^{-}\right)-f^{\prime \prime}\left(\xi^{+}\right) \overline{g^{\prime}}\left(\xi^{+}\right)+\int_{0}^{1}\left[g^{\prime \prime} \overline{f^{\prime \prime}}-f^{\prime \prime} \overline{g^{\prime \prime}}\right] \mathrm{d} x \\
& =\int_{0}^{1}\left[g^{\prime \prime} \overline{f^{\prime \prime}}-f^{\prime \prime} \overline{g^{\prime \prime}}\right] \mathrm{d} x+f^{\prime \prime}\left(\xi^{-}\right) \overline{\left\{-k(1-\xi)\left[(1-\gamma) f^{\prime \prime}\left(\xi^{-}\right)+\gamma f^{\prime \prime}\left(\xi^{+}\right)\right]-\frac{1}{k_{0}}\left[f^{\prime \prime}\left(\xi^{-}\right)-f^{\prime \prime}\left(\xi^{+}\right)\right]\right\}} \\
& -f^{\prime \prime}\left(\xi^{+}\right) \overline{\left\{k \xi\left[(1-\gamma) f^{\prime \prime}\left(\xi^{-}\right)+\gamma f^{\prime \prime}\left(\xi^{+}\right)\right]-\frac{1}{k_{0}}\left[f^{\prime \prime}\left(\xi^{-}\right)-f^{\prime \prime}\left(\xi^{+}\right)\right]\right\}} \\
& =\int_{0}^{1}\left[g^{\prime \prime} \overline{f^{\prime \prime}}-f^{\prime \prime} \overline{g^{\prime \prime}}\right] \mathrm{d} x-\left[k(1-\xi)(1-\gamma)+\frac{1}{k_{0}}\right]\left|f^{\prime \prime}\left(\xi^{-}\right)\right|^{2}-\left[k \xi \gamma+\frac{1}{k_{0}}\right]\left|f^{\prime \prime}\left(\xi^{+}\right)\right|^{2} \\
& -\left[k(1-\xi) \gamma-\frac{1}{k_{0}}\right] f^{\prime \prime}\left(\xi^{-}\right) \overline{f^{\prime \prime}\left(\xi^{+}\right)}-\left[k \xi(1-\gamma)-\frac{1}{k_{0}}\right] f^{\prime \prime}\left(\xi^{+}\right) \overline{f^{\prime \prime}\left(\xi^{-}\right)}
\end{aligned}
$$


SO

$$
\begin{aligned}
\operatorname{Re}\left\langle\mathcal{A}\left[\begin{array}{l}
f \\
g
\end{array}\right],\left[\begin{array}{l}
f \\
g
\end{array}\right]\right\rangle= & -\left[k(1-\xi)(1-\gamma)+\frac{1}{k_{0}}\right]\left|f^{\prime \prime}\left(\xi^{-}\right)\right|^{2}-\left[k \xi \gamma+\frac{1}{k_{0}}\right]\left|f^{\prime \prime}\left(\xi^{+}\right)\right|^{2} \\
& -\left[k(1-\xi) \gamma+k \xi(1-\gamma)-\frac{2}{k_{0}}\right] \operatorname{Re}\left(f^{\prime \prime}\left(\xi^{-}\right) \overline{f^{\prime \prime}\left(\xi^{+}\right)}\right) \\
\leq & -\left[k(1-\xi)(1-\gamma)+\frac{1}{k_{0}}\right]\left|f^{\prime \prime}\left(\xi^{-}\right)\right|^{2}-\left[k \xi \gamma+\frac{1}{k_{0}}\right]\left|f^{\prime \prime}\left(\xi^{+}\right)\right|^{2} \\
& +\left|k(1-\xi) \gamma+k \xi(1-\gamma)-\frac{2}{k_{0}}\right|\left|f^{\prime \prime}\left(\xi^{-}\right)\right|\left|f^{\prime \prime}\left(\xi^{+}\right)\right| \\
= & -\frac{1}{k_{0}}\left\langle\mathbb{A}\left[\begin{array}{l}
\left|f^{\prime \prime}\left(\xi^{-}\right)\right| \\
\left|f^{\prime \prime}\left(\xi^{+}\right)\right|
\end{array}\right],\left[\begin{array}{c}
\left|f^{\prime \prime}\left(\xi^{-}\right)\right| \\
\left|f^{\prime \prime}\left(\xi^{+}\right)\right|
\end{array}\right]\right\rangle_{\mathbb{R}^{2}},
\end{aligned}
$$

where $\mathbb{A}$ is a $2 \times 2$ symmetric real matrix:

$$
\mathbb{A}=\left[\begin{array}{cc}
k k_{0}(1-\xi)(1-\gamma)+1 & -\left|\frac{1}{2} k k_{0}(\xi+\gamma-2 \xi \gamma)-1\right| \\
-\left|\frac{1}{2} k k_{0}(\xi+\gamma-2 \xi \gamma)-1\right| & k k_{0} \xi \gamma+1
\end{array}\right]
$$

Therefore,

$$
\operatorname{Re}\left\langle\mathcal{A}\left[\begin{array}{l}
f \\
g
\end{array}\right],\left[\begin{array}{l}
f \\
g
\end{array}\right]\right\rangle \leq-\frac{1}{k_{0}}\left\langle\mathbb{A}\left[\begin{array}{l}
f^{\prime \prime}\left(\xi^{-}\right) \\
f^{\prime \prime}\left(\xi^{+}\right)
\end{array}\right],\left[\begin{array}{l}
f^{\prime \prime}\left(\xi^{-}\right) \\
f^{\prime \prime}\left(\xi^{+}\right)
\end{array}\right]\right\rangle_{\mathbb{R}^{2}} .
$$

Now, we show that $\mathbb{A}$ is nonnegative definite. This is equivalent to saying that both the trace and determinant of $\mathbb{A}$ are nonnegative. Indeed, since $k k_{0}(\xi-\gamma)^{2} \leq 4$ and

$$
(1-\xi)(\gamma-1) \leq \frac{(1-\xi+\gamma-1)^{2}}{4}=\frac{(\gamma-\xi)^{2}}{4} \leq \frac{1}{k k_{0}}
$$

or

$$
1+k k_{0}(1-\xi)(1-\gamma) \geq 0
$$

it follows that the trace of $\mathbb{A}$ is positive. Furthermore, it is computed that

$$
\begin{aligned}
\operatorname{det}(\mathbb{A})= & \left(k k_{0}(1-\xi)(1-\gamma)+1\right)\left(k k_{0} \xi \gamma+1\right)-\left(\frac{1}{2} k k_{0}(\xi+\gamma-2 \xi \gamma)-1\right)^{2} \\
= & 1+k k_{0}(1-\xi)(1-\gamma)+k k_{0} \xi \gamma+k^{2} k_{0}^{2} \xi \gamma(1-\xi)(1-\gamma) \\
& -1+k k_{0}(\xi+\gamma-2 \xi \gamma)-\frac{1}{4} k^{2} k_{0}^{2}(\xi+\gamma-2 \xi \gamma)^{2} \\
= & k k_{0}+k^{2} k_{0}^{2} \gamma \xi(1-\xi)(1-\gamma)-\frac{1}{4} k^{2} k_{0}^{2}(\xi+\gamma-2 \xi \gamma)^{2} \\
= & k k_{0}+\frac{1}{4} k^{2} k_{0}^{2}\left(4 \gamma \xi(1-\xi)(1-\gamma)-((1-\xi) \gamma+(1-\gamma) \xi)^{2}\right) \\
= & k k_{0}-\frac{1}{4} k^{2} k_{0}^{2}((1-\xi) \gamma-(1-\gamma) \xi)^{2} \\
= & \frac{1}{4} k k_{0}\left(4-k k_{0}(\xi-\gamma)^{2}\right) \geq 0 .
\end{aligned}
$$

Hence $\mathbb{A}$ is nonnegative definite. This fact together with (4.8) shows that $\mathcal{A}$ is dissipative:

$$
\operatorname{Re}\left\langle\mathcal{A}\left[\begin{array}{l}
f \\
g
\end{array}\right],\left[\begin{array}{l}
f \\
g
\end{array}\right]\right\rangle \leq 0, \forall(f, g) \in D(\mathcal{A}) .
$$


Since by Lemma 3.1, $\mathcal{A}^{-1}$ exists and is bounded, it follows from the Lumer-Phillips theorem ([23], Th. 4.3, p. 14) that $\mathcal{A}$ generates a $C_{0}$-semigroup of contractions on $\mathcal{H}$.

Next, suppose (3.2) is fulfilled. We show that there is no eigenvalue of $\mathcal{A}$ on the imaginary axis. Actually, since $\mathbb{A}$ is positive definite that is just justified, we may assume that $\lambda=i \tau^{2}, \tau>0$ is an eigenvalue of $\mathcal{A}$ such that $\mathcal{A}(f, g)=i \tau^{2}(f, g)$. It then follows from (4.8) that

$$
f^{\prime \prime}\left(\xi^{-}\right)=f^{\prime \prime}\left(\xi^{+}\right)=0 .
$$

In this case, (3.5) becomes

$$
\left\{\begin{array}{l}
f^{(4)}(x)+\alpha \tau^{4} f^{\prime \prime}(x)-\tau^{4} f(x)=0, x \in(0,1) \\
f(0)=f^{\prime \prime}(0)=f(1)=f^{\prime \prime}(1)=0 \\
f^{\prime \prime}(\xi)=0, f^{\prime}(\xi)=0
\end{array}\right.
$$

Let

$$
\tilde{\tau}_{1}=\sqrt{\frac{-\alpha \tau^{4}+\sqrt{\alpha^{2} \tau^{8}+4 \tau^{4}}}{2}}, \quad \tilde{\tau}_{2}=\sqrt{\frac{\alpha \tau^{4}+\sqrt{\alpha^{2} \tau^{8}+4 \tau^{4}}}{2}} .
$$

Both $\tilde{\tau}_{1}$ and $\tilde{\tau}_{2}$ are positive. By the condition $f(0)=f^{\prime \prime}(0)=0$, the solution of (4.10) can be represented as

$$
f(x)=c_{1} \sinh \tilde{\tau}_{1} x+c_{2} \sin \tilde{\tau}_{2} x
$$

for some constants $c_{1}, c_{2}$. By $f(1)=f^{\prime \prime}(1)=0$, we obtain

$$
\sin \tilde{\tau}_{2}=0 \text { or } \tilde{\tau}_{2}=n \pi, \quad n=0, \pm 1, \pm 2, \ldots,
$$

and hence

$$
f(x)=c_{2} \sin n \pi x .
$$

Since $f^{\prime}(\xi)=f^{\prime \prime}(\xi)=0$, it must have $c_{2}=0$. That is, there is only the zero solution to the equation (4.10). Therefore $\operatorname{Re}(\lambda)<0$ for any $\lambda \in \sigma(\mathcal{A})$. The proof is complete.

Proof of Theorem 3.4. From (3.6), (3.7) and (3.8), the general solutions of

$$
\left\{\begin{array}{l}
f^{(6)}(x)-\alpha \lambda^{2} f^{(4)}(x)+\lambda^{2} f^{\prime \prime}(x)=0, \\
f(0)=f^{\prime \prime}(0)=f^{(4)}(0)=f(1)=f^{\prime \prime}(1)=f^{(4)}(1)=0
\end{array}\right.
$$

are of the form

$$
f(x)=\left\{\begin{array}{l}
c_{1} x+c_{2} \sinh \tau_{1} x+c_{3} \sinh \tau_{2} x, x \in(0, \xi] \\
d_{1}(1-x)+d_{2} \sinh \tau_{1}(1-x)+d_{3} \sinh \tau_{2}(1-x), x \in(\xi, 1)
\end{array}\right.
$$

where $c_{i}, i=1,2, d_{j}, j=1,2,3$ are constants. Substitute other conditions of (3.6) into (4.12), to obtain

$$
\left\{\begin{array}{l}
c_{1} \xi+c_{2} a_{1}+c_{3} a_{2}-d_{1}(1-\xi)-d_{2} a_{3}-d_{3} a_{4}=0 \\
c_{1}+c_{2} \tau_{1} \hat{a}_{1}+c_{3} \tau_{2} \hat{a}_{2}+d_{1}+d_{2} \tau_{1} \hat{a}_{3}+d_{3} \tau_{2} \hat{a}_{4}=0 \\
c_{1} k_{0} \lambda+c_{2}\left(\tau_{1}^{2} a_{1}+k_{0} \lambda \tau_{1} \hat{a}_{1}\right)+c_{3}\left(\tau_{2}^{2} a_{2}+k_{0} \lambda \tau_{2} \hat{a}_{2}\right)-d_{2} \tau_{1}^{2} a_{3}-d_{3} \tau_{2}^{2} a_{4}=0 \\
c_{2}\left(\tau_{1}^{3} \hat{a}_{1}+\alpha k \lambda(1-\gamma) \tau_{1}^{2} a_{1}\right)+c_{3}\left(\tau_{2}^{3} \hat{a}_{2}+\alpha k \lambda(1-\gamma) \tau_{2}^{2} a_{2}\right) \\
+d_{2}\left(\tau_{1}^{3} \hat{a}_{3}+\alpha k \lambda \gamma \tau_{1}^{2} a_{3}\right)+d_{3}\left(\tau_{2}^{3} \hat{a}_{4}+\alpha k \lambda \gamma \tau_{2}^{2} a_{4}\right)=0 \\
c_{1} k_{0} \alpha \lambda^{3}+c_{2}\left(\tau_{1}^{4} a_{1}+k_{0} \alpha \lambda^{3} \tau_{1} \hat{a}_{1}\right)+c_{3}\left(\tau_{2}^{4} a_{2}+k_{0} \alpha \lambda^{3} \tau_{2} \hat{a}_{2}\right)-d_{2} \tau_{1}^{4} a_{3}-d_{3} \tau_{2}^{4} a_{4}=0 \\
c_{2}\left(\tau_{1}^{5} \hat{a}_{1}-\left(k \lambda-k \alpha^{2} \lambda^{3}\right)(1-\gamma) \tau_{1}^{2} a_{1}\right)+c_{3}\left(\tau_{2}^{5} \hat{a}_{2}-\left(k \lambda-k \alpha^{2} \lambda^{3}\right)(1-\gamma) \tau_{2}^{2} a_{2}\right) \\
+d_{2}\left(\tau_{1}^{5} \hat{a}_{3}-\left(k \lambda-k \alpha^{2} \lambda^{3}\right) \gamma \tau_{1}^{2} a_{3}\right)+d_{3}\left(\tau_{2}^{5} \hat{a}_{4}-\left(k \lambda-k \alpha^{2} \lambda^{3}\right) \gamma \tau_{2}^{2} a_{4}\right)=0
\end{array}\right.
$$


where

$$
\begin{aligned}
& a_{1}=\sinh \tau_{1} \xi, \hat{a}_{1}=\cosh \tau_{1} \xi, a_{2}=\sinh \tau_{2} \xi, \hat{a}_{2}=\cosh \tau_{2} \xi, \\
& a_{3}=\sinh \tau_{1}(1-\xi), \hat{a}_{3}=\cosh \tau_{1}(1-\xi), a_{4}=\sinh \tau_{2}(1-\xi), \hat{a}_{4}=\cosh \tau_{2}(1-\xi) .
\end{aligned}
$$

Write (4.13) to be

$$
\Delta(\lambda)\left(c_{1}, c_{2}, c_{3}, d_{1}, d_{2}, d_{3}\right)^{\top}=0
$$

where

$$
\Delta(\lambda)=\left[\Delta^{1}(\lambda), \Delta^{2}(\lambda)\right]
$$

with

$$
\begin{aligned}
\Delta^{1}(\lambda)=\left[\begin{array}{ccc}
\xi & a_{1} & a_{2} \\
1 & \tau_{1} \hat{a}_{1} & \tau_{2} \hat{a}_{2} \\
k_{0} \lambda & \tau_{1}^{2} a_{1}+k_{0} \lambda \tau_{1} \hat{a}_{1} & \tau_{2}^{2} a_{2}+k_{0} \lambda \tau_{2} \hat{a}_{2} \\
0 & \tau_{1}^{3} \hat{a}_{1}+\alpha k \lambda(1-\gamma) \tau_{1}^{2} a_{1} & \tau_{2}^{3} \hat{a}_{2}+\alpha k \lambda(1-\gamma) \tau_{2}^{2} a_{2} \\
k_{0} \alpha \lambda^{3} & \tau_{1}^{4} a_{1}+k_{0} \alpha \lambda^{3} \tau_{1} \hat{a}_{1} & \tau_{2}^{4} a_{2}+k_{0} \alpha \lambda^{3} \tau_{2} \hat{a}_{2} \\
0 & \tau_{1}^{5} \hat{a}_{1}-\left(k \lambda-k \alpha^{2} \lambda^{3}\right)(1-\gamma) \tau_{1}^{2} a_{1} & \tau_{2}^{5} \hat{a}_{2}-\left(k \lambda-k \alpha^{2} \lambda^{3}\right)(1-\gamma) \tau_{2}^{2} a_{2}
\end{array}\right], \\
\Delta^{2}(\lambda)=\left[\begin{array}{ccc}
-1+\xi & -a_{3} & -a_{4} \\
1 & \tau_{1} \hat{a}_{3} & \tau_{2} \hat{a}_{4} \\
0 & -\tau_{1}^{2} a_{3} & -\tau_{2}^{2} a_{4} \\
0 & \tau_{1}^{3} \hat{a}_{3}+\alpha k \lambda \gamma \tau_{1}^{2} a_{3} & \tau_{2}^{3} \hat{a}_{4}+\alpha k \lambda \gamma \tau_{2}^{2} a_{4} \\
0 & -\tau_{1}^{4} a_{3} & -\tau_{2}^{4} a_{4} \\
0 & \tau_{1}^{5} \hat{a}_{3}-\left(k \lambda-k \alpha^{2} \lambda^{3}\right) \gamma \tau_{1}^{2} a_{3} & \tau_{2}^{5} \hat{a}_{4}-\left(k \lambda-k \alpha^{2} \lambda^{3}\right) \gamma \tau_{2}^{2} a_{4}
\end{array}\right]
\end{aligned}
$$

Let $\tau_{1}, \tau_{2}$ be defined by (3.7). Then it is easy to show that as $|\lambda| \rightarrow \infty$,

$$
\begin{aligned}
& \tau_{1}(\lambda)=\frac{\sqrt{\alpha} \lambda}{\sqrt{2}} \sqrt{1+\sqrt{1-\frac{4}{\alpha^{2} \lambda^{2}}}}=\sqrt{\alpha} \lambda\left(1-\frac{1}{2 \alpha^{2} \lambda^{2}}+\mathcal{O}\left(\lambda^{-4}\right)\right) \\
& \tau_{2}(\lambda)=\sqrt{\frac{\alpha \lambda^{2}-\alpha \lambda^{2} \sqrt{1-\frac{4}{\alpha^{2} \lambda^{2}}}}{2}} \\
& =\sqrt{\frac{\alpha \lambda^{2}-\alpha \lambda^{2}\left(1-\frac{2}{\alpha^{2} \lambda^{2}}-\frac{2}{\alpha^{4} \lambda^{4}}+\mathcal{O}\left(\lambda^{-6}\right)\right.}{2}} \\
& =\sqrt{\frac{1}{\alpha}+\frac{1}{\alpha^{3} \lambda^{2}}+\mathcal{O}\left(\lambda^{-4}\right)}=\frac{1}{\sqrt{\alpha}}\left(1+\frac{1}{2 \alpha^{2} \lambda^{2}}+\mathcal{O}\left(\lambda^{-4}\right)\right) .
\end{aligned}
$$

Therefore

$$
\begin{aligned}
& a_{1}=\sinh \tau_{1} \xi=\sinh (\sqrt{\alpha} \lambda \xi)\left(1+\mathcal{O}\left(\lambda^{-2}\right)\right), \hat{a}_{1}=\cosh \tau_{1} \xi=\cosh (\sqrt{\alpha} \lambda \xi)\left(1+\mathcal{O}\left(\lambda^{-2}\right)\right) \\
& a_{2}=\sinh \tau_{2} \xi=\sinh \frac{\xi}{\sqrt{\alpha}}+\mathcal{O}\left(\lambda^{-2}\right), \hat{a}_{2}=\cosh \tau_{2} \xi=\cosh \frac{\xi}{\sqrt{\alpha}}+\mathcal{O}\left(\lambda^{-2}\right), \\
& a_{3}=\sinh \tau_{1}(1-\xi)=\sinh (\sqrt{\alpha} \lambda(1-\xi))\left(1+\mathcal{O}\left(\lambda^{-2}\right)\right), \\
& \hat{a}_{3}=\cosh \tau_{1}(1-\xi)=\cosh (\sqrt{\alpha} \lambda(1-\xi))\left(1+\mathcal{O}\left(\lambda^{-2}\right)\right), \\
& a_{4}=\sinh \tau_{2}(1-\xi)=\sinh \frac{1-\xi}{\sqrt{\alpha}}+\mathcal{O}\left(\lambda^{-2}\right), \hat{a}_{4}=\cosh \tau_{2}(1-\xi)=\cosh \frac{1-\xi}{\sqrt{\alpha}}+\mathcal{O}\left(\lambda^{-2}\right) .
\end{aligned}
$$

Furthermore, a direct computation shows that

$$
\operatorname{det}(\Delta(\lambda))=\operatorname{det}\left[\Delta^{11}(\lambda), \Delta^{12}(\lambda)\right]
$$


where

$$
\begin{aligned}
\Delta^{11}(\lambda) & =\left[\begin{array}{cc}
\tau_{1}^{2} a_{1}+k_{0} \lambda \tau_{1} \hat{a}_{1} \xi-k_{0} \lambda a_{1} & \tau_{2}^{2} a_{2}+k_{0} \lambda \tau_{2} \hat{a}_{2} \xi-k_{0} \lambda a_{2} \\
\tau_{1}^{3} \hat{a}_{1}+\alpha k \lambda(1-\gamma) \tau_{1}^{2} a_{1} & \tau_{2}^{3} \hat{a}_{2}+\alpha k \lambda(1-\gamma) \tau_{2}^{2} a_{2} \\
\tau_{1}^{4} a_{1}-\alpha \lambda^{2} \tau_{1}^{2} a_{1} & \tau_{2}^{4} a_{2}-\alpha \lambda^{2} \tau_{2}^{2} a_{2} \\
\tau_{1}^{5} \hat{a}_{1}-\alpha \lambda^{2} \tau_{1}^{3} \hat{a}_{1}-k \lambda(1-\gamma) \tau_{1}^{2} a_{1} & \tau_{2}^{5} \hat{a}_{2}-\alpha \lambda^{2} \tau_{2}^{3} \hat{a}_{2}-k \lambda(1-\gamma) \tau_{2}^{2} a_{2}
\end{array}\right], \\
\Delta^{12}(\lambda) & :=\left[\begin{array}{cc}
k_{0} \lambda a_{3}-\tau_{1}^{2} a_{3}-k_{0} \lambda \tau_{1} \hat{a}_{3}(1-\xi) & k_{0} \lambda a_{4}-\tau_{2}^{2} a_{4}-k_{0} \lambda \tau_{2} \hat{a}_{4}(1-\xi) \\
\tau_{1}^{3} \hat{a}_{3}+\alpha k \lambda \gamma \tau_{1}^{2} a_{3} & \tau_{2}^{3} \hat{a}_{4}+\alpha k \lambda \gamma \tau_{2}^{2} a_{4} \\
\alpha \lambda^{2} \tau_{1}^{2} a_{3}-\tau_{1}^{4} a_{3} & \alpha \lambda^{2} \tau_{2}^{2} a_{4}-\tau_{2}^{4} a_{4} \\
\tau_{1}^{5} \hat{a}_{3}-\alpha \lambda^{2} \tau_{1}^{3} \hat{a}_{3}-k \lambda \gamma \tau_{1}^{2} a_{3} & \tau_{2}^{5} \hat{a}_{4}-\alpha \lambda^{2} \tau_{2}^{3} \hat{a}_{4}-k \lambda \gamma \tau_{2}^{2} a_{4}
\end{array}\right] .
\end{aligned}
$$

Since

it follows that

$$
\tau_{1}^{2}-\alpha \lambda^{2}=-\frac{1}{\alpha}+\mathcal{O}\left(\lambda^{-2}\right), \quad \tau_{2}^{2}-\frac{1}{\alpha}=\mathcal{O}\left(\lambda^{-2}\right),
$$

$$
\begin{aligned}
\operatorname{det}(\Delta(\lambda)) & =-\lambda^{4} \tau_{1}^{4}\left|\begin{array}{cc}
a_{1}+\frac{k_{0}}{\sqrt{\alpha}} \hat{a}_{1} \xi+\mathcal{O}\left(\lambda^{-1}\right) & -a_{3}-\frac{k_{0}}{\sqrt{\alpha}} \hat{a}_{3}(1-\xi)+\mathcal{O}\left(\lambda^{-1}\right) \\
\sqrt{\alpha} \hat{a}_{1}+\alpha k(1-\gamma) a_{1}+\mathcal{O}\left(\lambda^{-2}\right) & \sqrt{\alpha} \hat{a}_{3}+\alpha k \gamma a_{3}+\mathcal{O}\left(\lambda^{-2}\right)
\end{array}\right| \\
& \times\left|\begin{array}{cc}
-\alpha \tau_{2}^{2} a_{2}+\mathcal{O}\left(\lambda^{-2}\right) & \alpha \tau_{2}^{2} a_{4}+\mathcal{O}\left(\lambda^{-2}\right) \\
-\alpha \tau_{2}^{3} \hat{a}_{2}+\mathcal{O}\left(\lambda^{-1}\right) & -\alpha \tau_{2}^{3} \hat{a}_{4}+\mathcal{O}\left(\lambda^{-1}\right)
\end{array}\right|
\end{aligned}
$$

and hence

$$
\begin{aligned}
& -\lambda^{-4} \tau_{1}^{-4} \operatorname{det}(\Delta(\lambda))=\mathcal{O}\left(\lambda^{-1}\right)+\left|\begin{array}{cc}
-\sinh \frac{\xi}{\sqrt{\alpha}} & \sinh \frac{1-\xi}{\sqrt{\alpha}} \\
-\frac{1}{\sqrt{\alpha}} \cosh \frac{\xi}{\sqrt{\alpha}} & -\frac{1}{\sqrt{\alpha}} \cosh \frac{1-\xi}{\sqrt{\alpha}}
\end{array}\right| \\
& \times\left|\begin{array}{cc}
\sinh (\sqrt{\alpha} \lambda \xi)+\frac{k_{0} \xi}{\sqrt{\alpha}} \cosh (\sqrt{\alpha} \lambda \xi) & -\sinh (\sqrt{\alpha} \lambda(1-\xi))-\frac{k_{0}(1-\xi)}{\sqrt{\alpha}} \cosh (\sqrt{\alpha} \lambda(1-\xi)) \\
\sqrt{\alpha} \cosh (\sqrt{\alpha} \lambda \xi)+\alpha k(1-\gamma) \sinh (\sqrt{\alpha} \lambda \xi) & \sqrt{\alpha} \cosh (\sqrt{\alpha} \lambda(1-\xi))+\alpha k \gamma \sinh (\sqrt{\alpha} \lambda(1-\xi))
\end{array}\right| .
\end{aligned}
$$

Finally, since

we obtain that

$$
\sinh \left(\frac{\xi}{\sqrt{\alpha}}+\frac{1-\xi}{\sqrt{\alpha}}\right)=\sinh \frac{\xi}{\sqrt{\alpha}} \cosh \frac{1-\xi}{\sqrt{\alpha}}+\cosh \frac{\xi}{\sqrt{\alpha}} \sinh \frac{1-\xi}{\sqrt{\alpha}}
$$

$$
\begin{aligned}
& -\frac{\lambda^{-4} \tau_{1}^{-4}}{\sinh \frac{1}{\sqrt{\alpha}}} \operatorname{det}(\Delta(\lambda))=\mathcal{O}\left(\lambda^{-1}\right) \\
& +\left|\begin{array}{cc}
\sinh (\sqrt{\alpha} \lambda \xi)+\frac{k_{0} \xi}{\sqrt{\alpha}} \cosh (\sqrt{\alpha} \lambda \xi) & -\sinh (\sqrt{\alpha} \lambda(1-\xi))-\frac{k_{0}(1-\xi)}{\sqrt{\alpha}} \cosh (\sqrt{\alpha} \lambda(1-\xi)) \\
\cosh (\sqrt{\alpha} \lambda \xi)+\sqrt{\alpha} k(1-\gamma) \sinh (\sqrt{\alpha} \lambda \xi) & \cosh (\sqrt{\alpha} \lambda(1-\xi))+\sqrt{\alpha} k \gamma \sinh (\sqrt{\alpha} \lambda(1-\xi))
\end{array}\right| .
\end{aligned}
$$

A further simplification gives

$$
\begin{aligned}
& \left|\begin{array}{cc}
\sinh (\sqrt{\alpha} \lambda \xi)+\frac{k_{0} \xi}{\sqrt{\alpha}} \cosh (\sqrt{\alpha} \lambda \xi) & -\sinh (\sqrt{\alpha} \lambda(1-\xi))-\frac{k_{0}(1-\xi)}{\sqrt{\alpha}} \cosh (\sqrt{\alpha} \lambda(1-\xi)) \\
\cosh (\sqrt{\alpha} \lambda \xi)+\sqrt{\alpha} k(1-\gamma) \sinh (\sqrt{\alpha} \lambda \xi) & \cosh (\sqrt{\alpha} \lambda(1-\xi))+\sqrt{\alpha} k \gamma \sinh (\sqrt{\alpha} \lambda(1-\xi))
\end{array}\right| \\
= & \left(1+\frac{k_{0} k \xi \gamma}{2}+\frac{k(1-\gamma) k_{0}(1-\xi)}{2}\right) \sinh (\sqrt{\alpha} \lambda)+\left(\frac{\sqrt{\alpha} k}{2}+\frac{k_{0}}{2 \sqrt{\alpha}}\right) \cosh (\sqrt{\alpha} \lambda) \\
& +\left(\frac{k_{0}}{2 \sqrt{\alpha}}-\frac{\sqrt{\alpha} k}{2}\right) \cosh (\sqrt{\alpha} \lambda(1-2 \xi))+\left(\frac{k_{0} k \xi \gamma}{2}-\frac{k(1-\gamma) k_{0}(1-\xi)}{2}\right) \sinh (\sqrt{\alpha} \lambda(1-2 \xi)) .
\end{aligned}
$$

Therefore, $\operatorname{det}(\Delta(\lambda))$ is represented as (3.9)-(3.10). The proof is complete. 
Proof of Corollary 3.5. Due to (3.9), it needs only to show that all zeros of $\Delta_{1}(\lambda)$ are located in some vertical strip paralleling to the imaginary axis in the complex plane. This is obvious because when $\operatorname{Re} \lambda \rightarrow+\infty$,

$$
\Delta_{1}(\lambda)=\frac{K_{1}+K_{2}}{2} \mathrm{e}^{\sqrt{\alpha} \lambda}[1+o(1)] \rightarrow \infty
$$

while $\operatorname{Re} \lambda \rightarrow-\infty$

$$
\Delta_{1}(\lambda)=\mathrm{e}^{-\sqrt{\alpha} \lambda}\left(\frac{K_{2}-K_{1}}{2}+o(1)\right)
$$

Let $\mathcal{A}_{0}$ be the operator $\mathcal{A}$ with $k=k_{0}=0$ in (2.10). Then $\mathcal{A}_{0}$ is skew-adjoint in $\mathcal{H}: \mathcal{A}_{0}^{*}=-\mathcal{A}_{0}$. Hence $\mathcal{A}_{0}$ generates a unitary-group on $\mathcal{H}$ and so

$$
\left\|R\left(\lambda, \mathcal{A}_{0}\right)\right\| \leq \frac{1}{|\lambda|}, \quad \forall \lambda \in \mathbb{C}, \operatorname{Re} \lambda \neq 0 .
$$

Lemma 4.2. For any $\lambda \in \rho\left(\mathcal{A}_{0}\right)$ and $(p, q) \in \mathcal{H},(\phi, \psi)=R\left(\lambda, \mathcal{A}_{0}\right)(p, q)$ is given by

$$
\left\{\begin{aligned}
\psi & =\lambda \phi-p \\
\phi & =\frac{q_{1}-\tau_{2}^{2} p_{1}}{\left(\tau_{1}^{2}-\tau_{2}^{2}\right) \sinh \tau_{1}} \sinh \tau_{1} x+\frac{p_{1} \tau_{1}^{2}-q_{1}}{\left(\tau_{1}^{2}-\tau_{2}^{2}\right) \sinh \tau_{2}} \sinh \tau_{2} x \\
& +\frac{1}{\tau_{1} \tau_{2}\left(\tau_{1}^{2}-\tau_{2}^{2}\right)} \int_{0}^{x}\left[\tau_{2} \sinh \tau_{1}(x-s)+\tau_{1} \sinh \tau_{2}(s-x)\right]\left[\lambda p+q-\alpha\left(\lambda p^{\prime \prime}+q^{\prime \prime}\right)\right] \mathrm{d} s,
\end{aligned}\right.
$$

where $\tau_{1}$ and $\tau_{2}$ are given by (3.7), and

$$
\left\{\begin{array}{l}
p_{1}:=\frac{-1}{\tau_{1} \tau_{2}\left(\tau_{1}^{2}-\tau_{2}^{2}\right)} \int_{0}^{1}\left[\tau_{2} \sinh \tau_{1}(1-s)+\tau_{1} \sinh \tau_{2}(s-1)\right]\left[\lambda p+q-\alpha\left(\lambda p^{\prime \prime}+q^{\prime \prime}\right)\right] \mathrm{d} s \\
q_{1}:=\frac{-1}{\left(\tau_{1}^{2}-\tau_{2}^{2}\right)} \int_{0}^{1}\left[\tau_{1} \sinh \tau_{1}(1-s)+\tau_{2} \sinh \tau_{2}(s-1)\right]\left[\lambda p+q-\alpha\left(\lambda p^{\prime \prime}+q^{\prime \prime}\right)\right] \mathrm{d} s .
\end{array}\right.
$$

Proof. Let $\lambda \in \sigma\left(\mathcal{A}_{0}\right)$ and $(p, q) \in \mathcal{H} .\left(\lambda-\mathcal{A}_{0}\right)(\phi, \psi)=(p, q)$ means that

$$
\left\{\begin{array}{l}
\lambda \phi-\psi=p, \quad \lambda \psi+\left(\mathcal{R} \frac{\mathrm{d}^{4}}{\mathrm{~d} x^{4}}\right) \phi=q \\
\phi(0)=\phi(1)=\phi^{\prime \prime}(0)=\phi^{\prime \prime}(1)=0
\end{array}\right.
$$

Hence $\psi=\lambda \phi-p$ and $\phi$ satisfies

$$
\left\{\begin{array}{l}
\phi^{(4)}+\lambda^{2} \phi-\alpha \lambda^{2} \phi^{\prime \prime}=\lambda p+q-\alpha\left(\lambda p^{\prime \prime}+q^{\prime \prime}\right), \\
\phi(0)=\phi(1)=\phi^{\prime \prime}(0)=\phi^{\prime \prime}(1)=0 .
\end{array}\right.
$$

Solve the first equation above with $\phi(0)=\phi^{\prime \prime}(0)=0$ to give

$$
\begin{aligned}
\phi(x) & =c_{1} \sinh \tau_{1} x+c_{2} \sinh \tau_{2} x+\frac{1}{\tau_{1} \tau_{2}\left(\tau_{1}^{2}-\tau_{2}^{2}\right)} \\
& \times \int_{0}^{x}\left[\tau_{2} \sinh \tau_{1}(x-s)+\tau_{1} \sinh \tau_{2}(s-x)\right]\left[\lambda p+q-\alpha\left(\lambda p^{\prime \prime}+q^{\prime \prime}\right)\right] \mathrm{d} s,
\end{aligned}
$$


where $c_{1}, c_{2}$ are constants to be determined so that $\phi^{\prime \prime}(0)=\phi^{\prime \prime}(1)=0$. This gives rise to

$$
\left\{\begin{array}{l}
c_{1} \sinh \tau_{1}+c_{2} \sinh \tau_{2}=p_{1}, \\
c_{1} \tau_{1}^{2} \sinh \tau_{1}+c_{2} \tau_{2}^{2} \sinh \tau_{2}=q_{1}
\end{array}\right.
$$

where $p_{1}$ and $q_{1}$ are given by (4.20). So

$$
c_{1}=\frac{q_{1}-\tau_{2}^{2} p_{1}}{\left(\tau_{1}^{2}-\tau_{2}^{2}\right) \sinh \tau_{1}}, \quad c_{2}=\frac{p_{1} \tau_{1}^{2}-q_{1}}{\left(\tau_{1}^{2}-\tau_{2}^{2}\right) \sinh \tau_{2}} .
$$

Substitute above into (4.21) to give (4.19). The proof is complete.

In order to prove the completeness of the root subspace, we need the following Theorem 4.3 [29].

Theorem 4.3. Let $\mathbf{A}$ be the generator of a $C_{0}$-semigroup in a Hilbert space $\mathbf{H}$. Assume that $\mathbf{A}$ is a discrete operator and for $\lambda \in \rho(\mathbf{A}), R(\lambda, \mathbf{A})$ is of the form

$$
R(\lambda, \mathbf{A}) Y=\frac{G(\lambda) Y}{F_{1}(\lambda)}, \forall Y \in \mathbf{H},
$$

where for each $Y \in \mathbf{H}, G(\lambda) Y$ is an $\mathbf{H}$-valued entire function with order less than or equal to $\rho_{1}$ and $F_{1}(\lambda)$ is a scalar entire function of order $\rho_{2}$. Let $\rho:=\max \left\{\rho_{1}, \rho_{2}\right\}<\infty$ and an integer $n$ so that $n-1 \leq \rho<n$. If there are $n+1$ rays $\gamma_{j}, j=0,1,2, \ldots, n$, on the complex plane

$$
\arg \gamma_{0}=\frac{\pi}{2}<\arg \gamma_{1}<\arg \gamma_{2}<\cdots<\arg \gamma_{n}=\frac{3 \pi}{2}
$$

with

$$
\arg \gamma_{j+1}-\arg \gamma_{j} \leq \frac{\pi}{n}, \quad 0 \leq j \leq n-1,
$$

so that for any $Y \in \mathbf{H}, R(\lambda, \mathbf{A}) Y$ is bounded on all rays $\gamma_{j}, 0<j<n$, as $|\lambda| \rightarrow \infty$, then $\operatorname{Sp}(\mathbf{A})=\mathbf{H}$.

Proof of Theorem 3.6. For any $(p, q) \in \mathcal{H}, \lambda \in \rho(\mathcal{A}) \cap \rho\left(\mathcal{A}_{0}\right)$, let

$$
(\phi, \psi)=R\left(\lambda, \mathcal{A}_{0}\right)(p, q), \quad(f, g)=R(\lambda, \mathcal{A})(p, q)-(\phi, \psi) .
$$

Then

$$
\left(\lambda-\mathcal{A}_{0}\right)(\phi, \psi)=(\lambda-\mathcal{A})[(f, g)+(\phi, \psi)]=(p, q) .
$$

So

Since

$$
\left\{\begin{array}{l}
\lambda f-g-b\left[g^{\prime}\right]_{\xi}-b\left[\psi^{\prime}\right]_{\xi}=0, \\
\lambda g+\left(\mathcal{R} \frac{\mathrm{d}^{4}}{\mathrm{~d} x^{4}}\right) f-\mathcal{R}\left(\frac{\mathrm{d}}{\mathrm{d} x} \delta_{\xi}\right)\left[f^{\prime \prime}\right]_{\xi}-\mathcal{R}\left(\frac{\mathrm{d}}{\mathrm{d} x} \delta_{\xi}\right)\left[\phi^{\prime \prime}\right]_{\xi}=0 .
\end{array}\right.
$$

it follows that

$$
\left[\psi^{\prime}\right]_{\xi}=\left[\phi^{\prime \prime}\right]_{\xi}=0, \quad\left[g^{\prime}\right]_{\xi}=k\left[(1-\gamma) f^{\prime \prime}\left(\xi^{-}\right)+\gamma f^{\prime \prime}\left(\xi^{+}\right)+\phi^{\prime \prime}(\xi)\right],
$$

$$
\left\{\begin{array}{l}
g=\lambda f-k b\left[(1-\gamma) f^{\prime \prime}\left(\xi^{-}\right)+\gamma f^{\prime \prime}\left(\xi^{+}\right)+\phi^{\prime \prime}(\xi)\right], \\
\left(\mathcal{R} \frac{\mathrm{d}^{4}}{\mathrm{~d} x^{4}}\right) f-\mathcal{R}\left(\frac{\mathrm{d}}{\mathrm{d} x} \delta_{\xi}\right)\left[f^{\prime \prime}\right]_{\xi}+\lambda^{2} f-k b \lambda\left[(1-\gamma) f^{\prime \prime}\left(\xi^{-}\right)+\gamma f^{\prime \prime}\left(\xi^{+}\right)+\phi^{\prime \prime}(\xi)\right]=0 .
\end{array}\right.
$$


By (4.19)

$$
\begin{aligned}
\phi^{\prime \prime}(\xi)= & \frac{\tau_{1}^{2}\left(q_{1}-\tau_{2}^{2} p_{1}\right)}{\left(\tau_{1}^{2}-\tau_{2}^{2}\right) \sinh \tau_{1}} \sinh \tau_{1} \xi+\frac{\tau_{2}^{2}\left(p_{1} \tau_{1}^{2}-q_{1}\right)}{\left(\tau_{1}^{2}-\tau_{2}^{2}\right) \sinh \tau_{2}} \sinh \tau_{2} \xi \\
& +\frac{1}{\left(\tau_{1}^{2}-\tau_{2}^{2}\right)} \int_{0}^{\xi}\left[\tau_{1} \sinh \tau_{1}(\xi-s)+\tau_{2} \sinh \tau_{2}(s-\xi)\right]\left[\lambda p+q-\alpha\left(\lambda p^{\prime \prime}+q^{\prime \prime}\right)\right] \mathrm{d} s
\end{aligned}
$$

This together with (4.15), (4.16) and (4.20) gives

$$
\begin{aligned}
\phi^{\prime \prime}(\xi)= & -\frac{\sinh \tau_{1} \xi}{\sqrt{\alpha} \sinh \tau_{1}} \int_{0}^{1} \sinh \tau_{1}(1-s)\left(p-\alpha p^{\prime \prime}\right) \mathrm{d} s \\
& +\frac{1}{\sqrt{\alpha}} \int_{0}^{\xi} \sinh \tau_{1}(\xi-s)\left(p-\alpha p^{\prime \prime}\right) \mathrm{d} s+\mathcal{O}\left(\lambda^{-1}\right) \text { as }|\lambda| \rightarrow \infty .
\end{aligned}
$$

Furthermore, $f$ satisfies the following equation:

$$
\left\{\begin{array}{l}
f^{(4)}(x)+\lambda^{2} f(x)-\alpha \lambda^{2} f^{\prime \prime}(x)=k b(x) \lambda\left[(1-\gamma) f^{\prime \prime}\left(\xi^{-}\right)+\gamma f^{\prime \prime}\left(\xi^{+}\right)+\phi^{\prime \prime}(\xi)\right], \\
f(0)=f(1)=f^{\prime \prime}(0)=f^{\prime \prime}(1)=0, \quad f\left(\xi^{-}\right)=f\left(\xi^{+}\right), \quad f^{\prime}\left(\xi^{-}\right)=f^{\prime}\left(\xi^{+}\right), \\
f^{\prime \prime}\left(\xi^{-}\right)-f^{\prime \prime}\left(\xi^{+}\right)=-\lambda k_{0} f^{\prime}(\xi), \\
f^{\prime \prime \prime}\left(\xi^{-}\right)-f^{\prime \prime \prime}\left(\xi^{+}\right)=-\alpha k \lambda\left[(1-\gamma) f^{\prime \prime}\left(\xi^{-}\right)+\gamma f^{\prime \prime}\left(\xi^{+}\right)+\phi^{\prime \prime}(\xi)\right],
\end{array}\right.
$$

which is equivalent to

$$
\left\{\begin{array}{l}
f^{(6)}(x)+\lambda^{2} f^{\prime \prime}(x)-\alpha \lambda^{2} f^{(4)}(x)=0, x \in(0,1), x \neq \xi \\
f(0)=f(1)=f^{\prime \prime}(0)=f^{\prime \prime}(1)=f^{(4)}(0)=f^{(4)}(1)=0, \\
f\left(\xi^{-}\right)=f\left(\xi^{+}\right), f^{\prime}\left(\xi^{-}\right)=f^{\prime}\left(\xi^{+}\right), \\
f^{\prime \prime}\left(\xi^{-}\right)-f^{\prime \prime}\left(\xi^{+}\right)=-\lambda k_{0} f^{\prime}(\xi), \\
f^{\prime \prime \prime}\left(\xi^{-}\right)-f^{\prime \prime \prime}\left(\xi^{+}\right)=-\alpha k \lambda\left[(1-\gamma) f^{\prime \prime}\left(\xi^{-}\right)+\gamma f^{\prime \prime}\left(\xi^{+}\right)+\phi^{\prime \prime}(\xi)\right], \\
f^{(4)}\left(\xi^{-}\right)-f^{(4)}\left(\xi^{+}\right)=-\alpha k_{0} \lambda^{3} f^{\prime}(\xi), \\
f^{(5)}\left(\xi^{-}\right)-f^{(5)}\left(\xi^{+}\right)=\left(k \lambda-k \alpha^{2} \lambda^{3}\right)\left[(1-\gamma) f^{\prime \prime}\left(\xi^{-}\right)+\gamma f^{\prime \prime}\left(\xi^{+}\right)+\phi^{\prime \prime}(\xi)\right] .
\end{array}\right.
$$

The solution of (4.26) is of the form (4.12) in which the coefficients satisfy

$$
\Delta(\lambda)\left(c_{1}, c_{2}, c_{3}, d_{1}, d_{2}, d_{3}\right)^{\top}=\Phi(\lambda)
$$

where $\Delta(\lambda)$ is defined by (4.14) and

$$
\Phi(\lambda):=\left(0,0,0,-\alpha k \lambda \phi^{\prime \prime}(\xi), 0,\left(k \lambda-k \alpha^{2} \lambda^{3}\right) \phi^{\prime \prime}(\xi)\right)^{\top} .
$$

Since $\lambda \in \rho(\mathcal{A}), \operatorname{det}(\Delta(\lambda)) \neq 0$ and (4.27) admits a unique solution:

$$
c_{i}=\frac{\operatorname{det}\left(\widetilde{\Delta}_{i}(\lambda)\right)}{\operatorname{det}(\Delta(\lambda))}, \quad d_{i}=\frac{\operatorname{det}\left(\widetilde{\Delta}_{i+3}(\lambda)\right)}{\operatorname{det}(\Delta(\lambda))}, \quad i=1,2,3,
$$


where $\widetilde{\Delta}_{i}(\rho), i=1,2, \ldots, 6$, are the matrices obtained by replacing the $i$ th-column of $\Delta(\lambda)$ with $\Phi(\lambda)$. Straightforward computations give

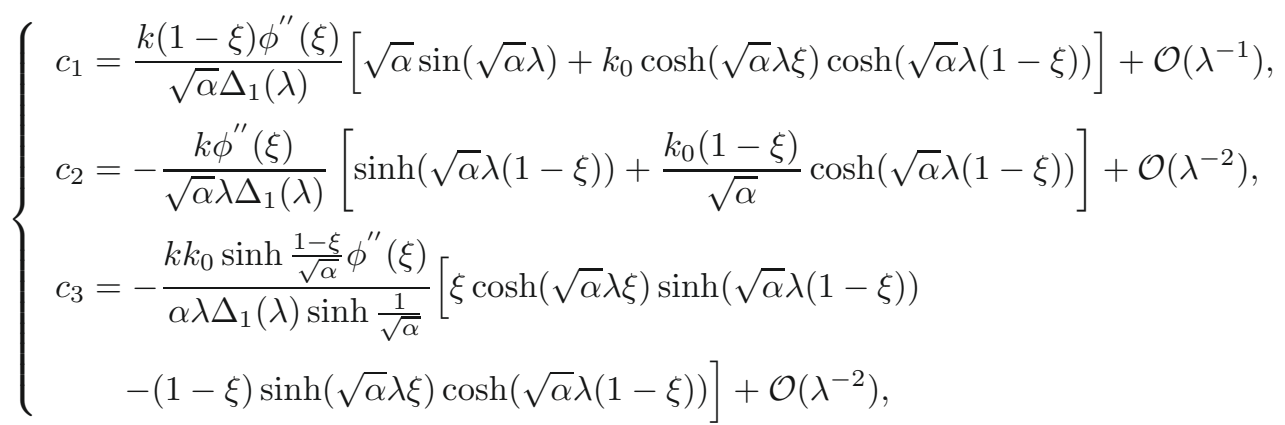

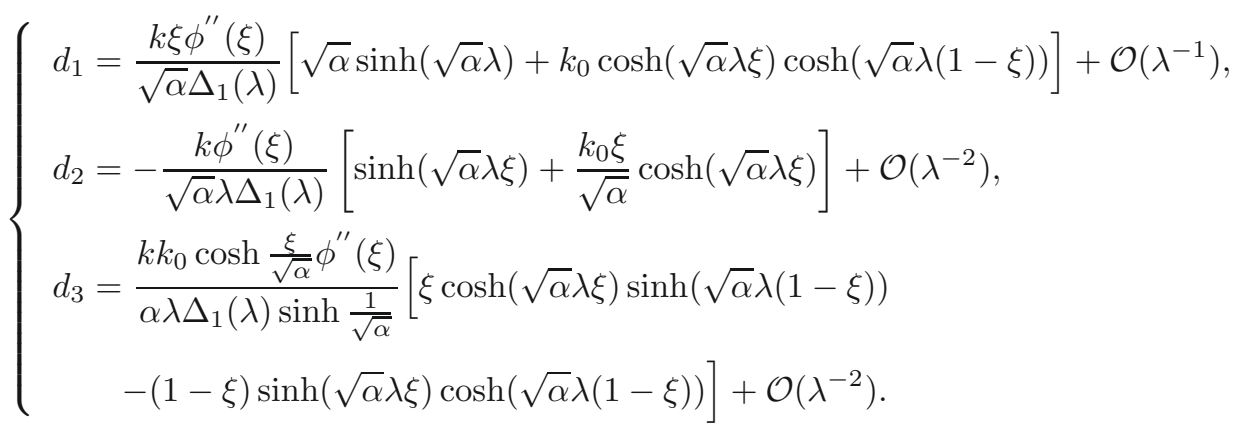

By (4.12),

$$
f^{\prime \prime}(x)=\left\{\begin{array}{l}
\tau_{1}^{2} c_{2} \sinh \tau_{1} x+\tau_{2}^{2} c_{3} \sinh \tau_{2} x, x \in(0, \xi], \\
\tau_{1}^{2} d_{2} \sinh \tau_{1}(1-x)+\tau_{2}^{2} d_{3} \sinh \tau_{2}(1-x), x \in(\xi, 1),
\end{array}\right.
$$

and so

$$
g^{\prime}(x)=\left\{\begin{array}{c}
c_{1} \lambda+\tau_{1} \lambda c_{2} \cosh \tau_{1} x+\tau_{2} \lambda c_{3} \cosh \tau_{2} x \\
-k(1-\xi)\left[(1-\gamma) f^{\prime \prime}\left(\xi^{-}\right)+\gamma f^{\prime \prime}\left(\xi^{+}\right)+\phi^{\prime \prime}(\xi)\right], \quad x \in(0, \xi], \\
-\lambda d_{1}-\tau_{1} \lambda d_{2} \cosh \tau_{1}(1-x)-\tau_{2} \lambda d_{3} \cosh \tau_{2}(1-x), \\
\quad+k \xi\left[(1-\gamma) f^{\prime \prime}\left(\xi^{-}\right)+\gamma f^{\prime \prime}\left(\xi^{+}\right)+\phi^{\prime \prime}(\xi)\right], \quad x \in(\xi, 1) .
\end{array}\right.
$$

Now, from (4.24) and (4.18), we have the following facts:

(a) there is a positive constant $M_{\xi}$ such that

$$
\left|\phi^{\prime \prime}(\xi)\right| \leq M_{\xi}\|p\|_{H^{2}(0,1) \cap H_{0}^{1}(0,1)} \leq M_{\xi}\|(p, q)\| \text { as } \operatorname{Re} \lambda \rightarrow-\infty ;
$$

(b) $\Delta_{1}(\lambda)=\mathrm{e}^{-\sqrt{\alpha} \lambda}\left(\frac{K_{2}-K_{1}}{2}+o(1)\right)$ as $\operatorname{Re} \lambda \rightarrow-\infty$ by (4.17) due to $K_{1} \neq K_{2}$;

(c) $\lim _{|\lambda| \rightarrow \infty}\left\|R\left(\lambda, \mathcal{A}_{0}\right)(p, q)\right\|=0$ and $\lim _{\operatorname{Re} \lambda \rightarrow-\infty}\left\|\lambda R\left(\lambda, \mathcal{A}_{0}\right)(p, q)\right\|<\infty$.

By these facts and (4.24), (4.30), (4.31), (4.32), (4.33), we see that $\|(f, g)\|=\left\|\left(f^{\prime \prime}, g^{\prime}\right)\right\|_{L^{2} \times L^{2}}$ is uniformly bounded as $\operatorname{Re} \lambda \rightarrow-\infty$. Since from (4.22),

$$
\|R(\lambda, \mathcal{A})(p, q)\| \leq\|(f, g)\|+\left\|R\left(\lambda, \mathcal{A}_{0}\right)(p, q)\right\|,
$$

it concludes that $\|R(\lambda, \mathcal{A})(p, q)\|$ is also uniformly bounded as $\operatorname{Re} \lambda \rightarrow-\infty$.

Finally, by (4.19) and (4.32)-(4.33),

$$
R(\lambda, \mathcal{A})(p, q)=(f, g)+(\phi, \psi)=\frac{G(\lambda ; p, q)}{F_{2}(\lambda)},
$$


where $G(\lambda ; p, q)$ is an $\mathcal{H}$-valued entire function with order less than or equal to 1 , and by (4.29), (4.32), (4.33), $F_{2}(\lambda)=p(\lambda) \operatorname{det}(\Delta(\lambda))$ is a scalar entire function of order 1 with polynomial $p(\lambda)$. Since $\sigma\left(\mathcal{A}_{0}\right)$ is a discrete set and (4.34) can be expanded analytically to $\sigma\left(\mathcal{A}_{0}\right) \cap \rho(A)$. So all assumptions of Theorem 4.3 are satisfied with $\rho=1, n=2, \gamma_{1}=\{\lambda \mid \arg \lambda=\pi\}$. Therefore $\operatorname{Sp}(\mathcal{A})=\mathcal{H}$.

Let us recall that a set $\Pi=\left\{a_{\alpha}, \alpha \in \Upsilon\right\} \subset \mathbb{R}^{2}$ is called separated if $\inf _{\alpha, \beta \in \Upsilon}\left|a_{\alpha}-a_{\beta}\right|>0$. Let $\Omega=\left\{\nu_{k}\right\}_{k \in \mathcal{J}}$ be a sequence of $\mathbb{C}$ satisfying $\left|\operatorname{Re} \nu_{k}\right|<\infty$. Suppose each $\nu_{k}$ appears in $\Omega$ at most finite times and $\Omega$ has no finite accumulation points. Then $\Omega$ can be ordered in such a way that $\left\{\operatorname{Im} \nu_{k}\right\}$ form a nondecreasing sequence. Suppose further that each $\nu_{k}$ is repeated in a number of time of its appearance in $\Omega$, and $\Omega$ is a union of $M$ separable sets $\left\{\widetilde{\Omega}_{\ell}\right\}: \Omega=\bigcup_{\ell=1}^{M} \widetilde{\Omega}_{\ell}$. Define

$$
D^{+}(\Omega)=\lim _{r \rightarrow \infty} \frac{n^{+}(r)}{r}
$$

where

Then [17]

$$
n^{+}(r)=\sup _{x \in \mathbb{R}}\{\text { the number of } \operatorname{Im}(\Omega) \cap[x, x+r)\} .
$$

$$
D^{+}(\Omega)<\infty .
$$

An entire function $F(\cdot)$ is said to be of exponential type if the inequality

$$
|F(z)| \leq C \mathrm{e}^{L|z|}
$$

holds for some positive constants $C$ and $L$ and all complex values of $z$ [31]. A point $z_{0} \in \mathbb{C}$ such that $F\left(z_{0}\right)=0$ is called a zero of the entire function $F$. The integer $\ell$ such that $F\left(z_{0}\right)=F^{\prime}\left(z_{0}\right)=\cdots=F^{(\ell)}\left(z_{0}\right)=0$ but $F^{(\ell+1)}\left(z_{0}\right) \neq 0$ is called the vanishing order of $F$. We say $z_{0}$ is a simple zero of $F$ if $\ell=0$, otherwise, it is called a multiple zero. An entire function of exponential type $F$ is said to be of sine-type if (see Def. II.1.27 of [3]):

(a) the zeros of $F$ lie in a strip $\{z \in \mathbb{C}|| \operatorname{Re} z \mid \leq c\}$ for some $c>0$;

(b) there exist constants $c_{1}, c_{2}>0$ and $x_{0} \in \mathbb{R}$ such that $c_{1} \leq\left|F\left(x_{0}+i y\right)\right| \leq c_{2}$ for all $y \in \mathbb{R}$.

The class of sine-type functions was first introduced in [19] to deal with problems of interpolation by entire functions and Riesz basis property of the sets of complex exponentials in $L^{2}$ space. The distribution of the zeros of sine-type function is characterized by the following remarkable Proposition 4.4 (see Prop. II.1.28 of [3]).

Proposition 4.4. Let $F$ be a sine-type function. Then the set of its zeros (a multiple zero is repeated in a number of times of its vanishing order) is a finite union of separable sets, that is, there exists an integer $M>0$ such that

$$
\text { zeros of } F=\bigcup_{i=1}^{M} \tilde{\Lambda}_{i}, \inf _{p \neq q, \nu_{p}^{i}, \nu_{q}^{i} \in \tilde{\Lambda}_{i}}\left|\nu_{p}^{i}-\nu_{q}^{i}\right|>0 .
$$

Consequently, the vanishing orders of a sine-type function at its zeros must be uniformly bounded.

Proof of Theorem 3.7. Let $\Delta_{1}(\lambda)$ be defined by (3.10), which is obviously an entire function of exponential type. First, it is seen by (4.9) that $K_{1}>0, K_{2}>0$ in (3.10). Secondly, from the proof of Corollary 3.5,

$$
\Delta_{1}(\lambda)=\frac{K_{1}+K_{2}}{2} \mathrm{e}^{\sqrt{\alpha} \lambda}[1+o(1)] \rightarrow \infty \text { as } \operatorname{Re} \lambda \rightarrow+\infty
$$

This together with Corollary 3.5 shows that $\Delta_{1}(\lambda)$ is a sine-type function. On the other hand, it follows from (3.9) that the zeros of $\operatorname{det}(\Delta(\lambda))$ approach those of $\Delta_{1}(\lambda)$. By the Rouché's theorem, we can say that

$$
\text { zeros of } \operatorname{det}(\Delta(\lambda))=\bigcup_{i=1}^{K_{0}} \Omega_{i}, \inf _{p \neq q, \lambda_{i}^{p}, \lambda_{i}^{q} \in \Omega_{i}}\left|\lambda_{i}^{p}-\lambda_{i}^{q}\right|>0
$$


where $K_{0}>0$ is an integer and a multiple zero is repeated in a number of times of its vanishing order. This implies particularly that all vanishing orders of zeros of $\operatorname{det}(\Delta(\lambda))$ are uniformly bounded.

Now from (3.6), (4.12), for each eigenvalue $\lambda$ of $\mathcal{A}$, its geometrical multiplicity is less than 6 . On the other hand, it follows from a general formula of [22], p. 148, that

$$
m_{(a)}(\lambda) \leq p_{\lambda} \cdot m_{(g)}(\lambda)
$$

where $p_{\lambda}$ is the order of pole of $R(\lambda, \mathcal{A})$ at $\lambda$. The expression (4.34) asserts that $p_{\lambda}$ does not exceed the vanishing order of $\operatorname{det}(\Delta(\lambda))$ at $\lambda$. Therefore

$$
\sup _{\lambda \in \sigma(\mathcal{A})} m_{(a)}(\lambda)<\infty .
$$

Denote $\sigma(\mathcal{A})=\left\{\lambda_{n}\right\}_{n \in \mathcal{J}}$. Since each $\lambda_{n}$ is of algebraic multiplicity $m_{a}\left(\lambda_{n}\right)$, we have a set of complex exponentials in terms of the eigenvalues of $\mathcal{A}$ :

$$
E_{n}(t)=\left\{\mathrm{e}^{\lambda_{n} t}, t \mathrm{e}^{\lambda_{n} t}, \ldots, t^{m_{a}\left(\lambda_{n}\right)-1} \mathrm{e}^{\lambda_{n} t}\right\}, \quad n \in \mathcal{J} .
$$

By (4.37) and (4.38), the eigenvalues of $\mathcal{A}$ can decompose into a finite union of separable sets (a multiple eigenvalue is repeated in a number of time of its algebraic multiplicity).

$$
\text { eigenvalues of } \mathcal{A}=\Lambda=\bigcup_{n=1}^{N} \Lambda_{n}, \inf _{i \neq j, \lambda_{i}, \lambda_{j} \in \Lambda_{n}}\left|\lambda_{i}-\lambda_{j}\right|>0, \quad \forall 1 \leq n \leq N .
$$

Let $\delta=\min _{1 \leq n \leq N} \inf _{i \neq j, \lambda_{i}, \lambda_{j} \in \Lambda_{n}}\left|\lambda_{i}-\lambda_{j}\right|>0$. Then for any $r<r_{0}=\delta /(2 N)$, by the discussions in Section 3 of [18], there exist $\Lambda^{p}=\left\{\lambda_{j}^{p}\right\}_{j=1}^{N^{p}}, N^{p} \leq N, p \in \mathcal{J}$, the $p$-th connected component of intersection of $\Lambda$ with $\bigcup_{n \in \mathcal{J}} D_{\lambda_{n}}(r)$, where $D_{\lambda_{n}}(r)$ is a disk with center $\lambda_{n}$ and radius $r$, such that

$$
\sigma(\mathcal{A})=\bigcup_{p \in \mathcal{J}} \Lambda^{p}
$$

We may assume without loss of generality that $\left\{\lambda_{n}\right\}$ are arranged for $\operatorname{Im} \lambda_{n}$ to be nondecreasing for each $p \in \mathcal{J}$ and $\operatorname{Re} \lambda_{1}^{p} \geq \operatorname{Re} \lambda_{2}^{p} \geq \cdots \geq \operatorname{Re} \lambda_{p}^{N^{p}}$. Construct a family of the generalized divided difference (GDD) of the following $[4,18]$ :

$$
E^{p}(\Lambda, r)=\left\{\left[\lambda_{1}^{p}\right](t),\left[\lambda_{1}^{p}, \lambda_{2}^{p}\right](t), \ldots,\left[\lambda_{1}^{p}, \lambda_{2}^{p}, \ldots, \lambda_{p}^{N^{p}}\right](t)\right\}, p \in \mathcal{J} .
$$

By (4.35), $D^{+}(\Lambda)<\infty$. According to Proposition 3.2 of [18], for any $T>2 \pi D^{+}(\Lambda)$, the family of GDD $\left\{E^{p}(\Lambda, r)\right\}_{p \in \mathcal{J}}$ form a Riesz basis for the closed subspace spanned by itself in $L^{2}(0, T)$. Since $N^{p} \leq N$, all conditions of Theorem 3.1 of [18] are satisfied. This together with $\operatorname{Sp}(\mathcal{A})=\mathcal{H}$ claimed by Theorem 3.6, concludes the assertions. The proof is complete.

Lemma 4.5. If $1+k k_{0}(1-\gamma)(1-\xi)>0$, then $\Delta_{1}(i \eta) \neq 0$ for any $\eta \in \mathbb{R}$.

Proof. Let $\lambda=i s$ with $s \in \mathbb{R}$ be a zero of $\Delta_{1}(\lambda)$. Then

$$
\Delta_{1}(s)=K_{2} \cos (\sqrt{\alpha} s)+i K_{1} \sin (\sqrt{\alpha} s)+K_{3} \cos (\sqrt{\alpha} s(1-2 \xi))+i K_{4} \sin (\sqrt{\alpha} s(1-2 \xi))=0,
$$

which can be decomposed into

$$
\left\{\begin{array}{l}
K_{2} \cos (\sqrt{\alpha} s)+K_{3} \cos (\sqrt{\alpha} s(1-2 \xi))=0 \\
K_{1} \sin (\sqrt{\alpha} s)+K_{4} \sin (\sqrt{\alpha} s(1-2 \xi))=0
\end{array}\right.
$$


or

$$
\left\{\begin{array}{l}
\cos (\sqrt{\alpha} s)\left(K_{2}+K_{3} \cos (2 s \xi \sqrt{\alpha})\right)+K_{3} \sin (\sqrt{\alpha} s) \sin (2 s \xi \sqrt{\alpha})=0 \\
\sin (\sqrt{\alpha} s)\left(K_{1}+K_{4} \cos (2 s \xi \sqrt{\alpha})\right)-K_{4} \cos (\sqrt{\alpha} s) \sin (2 s \xi \sqrt{\alpha})=0 .
\end{array}\right.
$$

When $\sin (\sqrt{\alpha} s)=0, \cos (\sqrt{\alpha} s)=1$ or -1 . There are two cases:

Case 1. $K_{4}=0$. In this case, $\cos (\sqrt{\alpha} s(1-2 \xi))= \pm \frac{K_{2}}{K_{3}}$, which contradicts the fact that $0 \leq\left|K_{3}\right|<K_{2}$.

Case 2. $\sin (2 s \xi \sqrt{\alpha})=0$. In this case, $\cos (2 s \xi \sqrt{\alpha})=1$ or -1 . Hence

$$
K_{2}+K_{3}=\frac{k_{0}}{\sqrt{\alpha}}=0 \quad \text { or } \quad K_{2}-K_{3}=\sqrt{\alpha} k=0
$$

which contradicts the fact that $k_{0}, k>0$. Thus, $\sin (\sqrt{\alpha} s) \neq 0$.

When $K_{1}+K_{4} \cos (2 s \xi \sqrt{\alpha})=0$, there are also two cases:

Case 1. $K_{4}=0$. In this case, $K_{1}=0$. But this does not happen because $K_{4}=0$ means that

$$
\frac{k_{0} k \xi \gamma}{2}=\frac{k(1-\gamma) k_{0}(1-\xi)}{2}
$$

and so $K_{1}=1+k k_{0} \xi \gamma \neq 0$.

Case 2. $K_{4} \neq 0$. In this case, $\cos (2 s \xi \sqrt{\alpha})=-K_{1} / K_{4}$ and so $\left|K_{1}\right| \leq\left|K_{4}\right|$, which contradicts the fact that $k k_{0}(\gamma-1)(1-\xi)<1$.

Therefore, it always has $K_{1}+K_{4} \cos (2 s \xi \sqrt{\alpha}) \neq 0$. Furthermore, from (4.42) and the fact $\operatorname{that} \cos (\sqrt{\alpha} s) \neq 0$, we have

or

$$
K_{2}+K_{3} \cos (2 s \xi \sqrt{\alpha})+\frac{K_{3} K_{4} \sin ^{2}(2 s \xi \sqrt{\alpha})}{K_{1}+K_{4} \cos (2 s \xi \sqrt{\alpha})}=0
$$

$$
\left(K_{2}+K_{3} \cos (2 s \xi \sqrt{\alpha})\right)\left(K_{1}+K_{4} \cos (2 s \xi \sqrt{\alpha})\right)+K_{3} K_{4} \sin ^{2}(2 s \xi \sqrt{\alpha})=0 .
$$

So we only need to find the solutions of the following equation

$$
K_{1} K_{2}+K_{3} K_{4}+\left(K_{1} K_{3}+K_{2} K_{4}\right) \cos (2 s \xi \sqrt{\alpha})=0
$$

To do this, we notice that

$$
\begin{aligned}
K_{1} K_{3}+K_{2} K_{4}= & \left(1+\frac{k_{0} k \xi \gamma}{2}+\frac{k(1-\gamma) k_{0}(1-\xi)}{2}\right)\left(\frac{k_{0}}{2 \sqrt{\alpha}}-\frac{\sqrt{\alpha} k}{2}\right) \\
& +\left(\frac{\sqrt{\alpha} k}{2}+\frac{k_{0}}{2 \sqrt{\alpha}}\right)\left(\frac{k_{0} k \xi \gamma}{2}-\frac{k(1-\gamma) k_{0}(1-\xi)}{2}\right) \\
= & \frac{k_{0}}{2 \sqrt{\alpha}}-\frac{\sqrt{\alpha} k}{2}+\frac{k_{0} k \xi \gamma}{2} \frac{k_{0}}{\sqrt{\alpha}}-k(1-\gamma) k_{0}(1-\xi) \frac{\sqrt{\alpha} k}{2}, \\
K_{1} K_{2}+K_{3} K_{4}= & \left(1+\frac{k_{0} k \xi \gamma}{2}+\frac{k(1-\gamma) k_{0}(1-\xi)}{2}\right)\left(\frac{\sqrt{\alpha} k}{2}+\frac{k_{0}}{2 \sqrt{\alpha}}\right) \\
& +\left(\frac{k_{0}}{2 \sqrt{\alpha}}-\frac{\sqrt{\alpha} k}{2}\right)\left(\frac{k_{0} k \xi \gamma}{2}-\frac{k(1-\gamma) k_{0}(1-\xi)}{2}\right) \\
= & \frac{\sqrt{\alpha} k}{2}+\frac{k_{0}}{2 \sqrt{\alpha}}+\frac{k_{0}}{\sqrt{\alpha}} \frac{k_{0} k \xi \gamma}{2}+\sqrt{\alpha} k \frac{k(1-\gamma) k_{0}(1-\xi)}{2} .
\end{aligned}
$$


Since $k k_{0}(\gamma-1)(1-\xi)<1$, it follows that

$$
\begin{gathered}
K_{1} K_{2}+K_{3} K_{4}-\left(K_{1} K_{3}+K_{2} K_{4}\right)=\sqrt{\alpha} k\left(1+k k_{0}(1-\gamma)(1-\xi)\right)>0, \\
K_{1} K_{2}+K_{3} K_{4}+\left(K_{1} K_{3}+K_{2} K_{4}\right)=\frac{k_{0}}{\sqrt{\alpha}}\left(1+k_{0} k \xi \gamma\right)>0 .
\end{gathered}
$$

Hence

$$
\left|K_{1} K_{3}+K_{2} K_{4}\right|<K_{1} K_{2}+K_{3} K_{4}
$$

This shows that (4.43) has no solution. Therefore there is no zero for $\Delta_{1}(\lambda)$ on the imaginary axis. The proof is complete.

Lemma 4.6. If $1+k k_{0}(1-\gamma)(1-\xi)>0$, then the imaginary axis is not the asymptote of the zeros of $\Delta_{1}(\lambda)$. Proof. We only need to show that $\inf _{s \in \mathbb{R}}\left|\Delta_{1}(i s)\right|>0$. This will be accomplished by arguments of contradiction. Assume that

$$
\lim _{n \rightarrow \infty}\left|G\left(i s_{n}\right)\right|=0 \text { as }\left|s_{n}\right| \rightarrow \infty, \quad s_{n} \in \mathbb{R} .
$$

Then it follows from (4.41) that as $n \rightarrow \infty$

$$
\left\{\begin{array}{c}
e_{n}:=K_{2} \cos \left(\sqrt{\alpha} s_{n}\right)+K_{3} \cos \left(\sqrt{\alpha} s_{n}(1-2 \xi)\right) \rightarrow 0, \\
f_{n}:=K_{1} \sin \left(\sqrt{\alpha} s_{n}\right)+K_{4} \sin \left(\sqrt{\alpha} s_{n}(1-2 \xi)\right) \rightarrow 0 .
\end{array}\right.
$$

On the other hand, simple computations give

$$
\begin{aligned}
& \cos \left(\sqrt{\alpha} s_{n}\right)=\frac{\left(K_{1}+K_{4} \cos \left(2 s_{n} \xi \sqrt{\alpha}\right)\right) e_{n}-K_{3} \sin \left(2 s_{n} \xi \sqrt{\alpha}\right) f_{n}}{K_{1} K_{2}+K_{3} K_{4}+\left(K_{1} K_{3}+K_{2} K_{4}\right) \cos \left(2 s_{n} \xi \sqrt{\alpha}\right)}, \\
& \sin \left(\sqrt{\alpha} s_{n}\right)=\frac{K_{4} \sin \left(2 s_{n} \xi \sqrt{\alpha}\right) e_{n}+\left(K_{2}+K_{3} \cos \left(2 s_{n} \xi \sqrt{\alpha}\right)\right) f_{n}}{K_{1} K_{2}+K_{3} K_{4}+\left(K_{1} K_{3}+K_{2} K_{4}\right) \cos \left(2 s_{n} \xi \sqrt{\alpha}\right)} .
\end{aligned}
$$

In terms of (4.44) and (4.45), we have

$$
0<K_{5} \leq K_{1} K_{2}+K_{3} K_{4}+\left(K_{1} K_{3}+K_{2} K_{4}\right) \cos \left(2 s_{n} \xi \sqrt{\alpha}\right) \leq K_{6},
$$

where

$$
\begin{aligned}
& K_{5}:=\min \left\{\sqrt{\alpha} k\left(1+k k_{0}(1-\gamma)(1-\xi)\right), \frac{k_{0}}{\sqrt{\alpha}}\left(1+k_{0} k \xi \gamma\right)\right\}, \\
& K_{6}:=\max \left\{\sqrt{\alpha} k\left(1+k k_{0}(1-\gamma)(1-\xi)\right), \frac{k_{0}}{\sqrt{\alpha}}\left(1+k_{0} k \xi \gamma\right)\right\} .
\end{aligned}
$$

By virtue of (4.47) and (4.48), $\cos \left(\sqrt{\alpha} s_{n}\right) \rightarrow 0, \sin \left(\sqrt{\alpha} s_{n}\right) \rightarrow 0$ as $n \rightarrow \infty$, a contradiction. Therefore, $\inf _{s \in \mathbb{R}}\left|\Delta_{1}(i s)\right|>0$.

Proof of Theorem 3.8. Looking back (4.9), we see that under the condition (3.2),

$$
1+k k_{0}(1-\gamma)(1-\xi)>0 .
$$

So, the required result is a direct consequence of Lemma 4.6 by applying the Rouché's theorem and (3.9). 
Proof of Theorem 3.10. Under the condition, it follows from Remark 3.9 that $1+k k_{0}(1-\gamma)(1-\xi)=0$. In this case $K_{1}=K_{4}$ in (3.10). Let $\lambda=i s, s \in \mathbb{R}$. If we assign

$$
\cos (2 s \xi \sqrt{\alpha})=-1, \cos (s \sqrt{\alpha})=0
$$

then (4.41) has solution, that is, $\Delta_{1}(i s)=0$. Now the solutions of (4.49) are

$$
s_{n_{1}}=\frac{n_{1} \pi+\pi / 2}{\xi \sqrt{\alpha}}, s_{n_{2}}=\frac{n_{2} \pi+\pi / 2}{\sqrt{\alpha}}, n_{1}, n_{2} \in \mathbb{Z}
$$

Set $s_{n_{1}}=s_{n_{2}}$ to get $\xi=\frac{n_{1}+1 / 2}{n_{2}+1 / 2}$. Take $n_{2}=2, n_{1}=1$. Then $\xi=\frac{n_{1}+1 / 2}{n_{2}+1 / 2}=\frac{3}{5} \in(0,1)$. Solve the equation

$$
\frac{n_{1}+1 / 2}{n_{2}+1 / 2}=\frac{3}{5},
$$

to get

$$
n_{1}=3 m+1, n_{2}=2+5 m
$$

for all integers $m>0$. That is, when $\xi=\frac{3}{5}$, all $s_{m}=\frac{(2+5 m) \pi+\pi / 2}{\sqrt{\alpha}}$ satisfy $\Delta_{1}\left(i s_{m}\right)=0$. Since $s_{m} \rightarrow \infty$ as $m \rightarrow+\infty$, we see, from (3.9), that the imaginary axis is the asymptote of eigenvalues of $\mathcal{A}$. So the system (2.14) is not exponentially stable when $\xi=\frac{3}{5}$.

\section{REFERENCES}

[1] K. Ammari and M. Tucsnak, Stabilization of Bernoulli-Euler beams by means of a pointwise feedback force. SIAM J. Control Optim. 39 (2000) 1160-1181.

[2] K. Ammari, Z. Liu and M. Tucsnak, Decay rates for a beam with pointwise force and moment feedback. Math. Control Signals Systems 15 (2002) 229-255.

[3] S.A. Avdonin and S.A. Ivanov, Families of Exponentials: The Method of Moments in Controllability Problems for Distributed Parameter Systems. Cambridge University Press, Cambridge, UK (1995).

[4] S.A. Avdonin and S.A. Ivanov, Riesz bases of exponentials and divided differences. St. Petersburg Math. J. 13 (2002) $339-351$.

[5] S.A. Avdonin and W. Moran, Simultaneous control problems for systems of elastic strings and beams. Syst. Control Lett. 44 (2001) $147-155$.

[6] C. Castro and E. Zuazua, A hybrid system consisting of two flexible beams connected by a point mass: spectral analysis and well-posedness in asymmetric spaces. ESAIM: Proc. 2 (1997) 17-53.

[7] C. Castro and E. Zuazua, Boundary controllability of a hybrid system consisting in two flexible beams connected by a point mass. SIAM J. Control Optim. 36 (1998) 1576-1595.

[8] C. Castro and E. Zuazua, Exact boundary controllability of two Euler-Bernoulli beams connected by a point mass. Math Comput. Modelling 32 (2000) 955-969.

[9] G. Chen, M.C. Delfour, A.M. Krall and G. Payre, Modeling, stabilization and control of serially connected beams. SIAM J. Control Optim. 25 (1987) 526-546.

[10] G. Chen, S.G. Krantz, D.L. Russell, C.E. Wayne, H.H. West and M.P. Coleman, Analysis, designs, and behavior of dissipative joints for coupled beams. SIAM J. Appl. Math. 49 (1989) 1665-1693.

[11] S. Cox and E. Zuazua, The rate at which energy decays in a damped string. Comm. Partial Diff. Eq. 19 (1994) $213-243$.

[12] S. Cox and E. Zuazua, The rate at which energy decays in a string damped at one end. Indiana Univ. Math. J. 44 (1995) $545-573$.

[13] R.F. Curtain and G. Weiss, Exponential stabilization of well-posed systems by colocated feedback. SIAM J. Control Optim. 45 (2006) 273-297.

[14] R. Dáger and E. Zuazua, Wave Propagation, Observation and Control in 1-d Flexible Multi-Structures, Mathématiques et Applications 50. Springer-Verlag, Berlin (2006).

[15] B.Z. Guo and K.Y. Chan, Riesz basis generation, eigenvalues distribution, and exponential stability for a Euler-Bernoulli beam with joint feedback control. Rev. Mat. Complut. 14 (2001) 205-229.

[16] B.Z. Guo and J.M. Wang, Riesz basis generation of an abstract second-order partial differential equation system with general non-separated boundary conditions. Numer. Funct. Anal. Optim. 27 (2006) 291-328.

[17] B.Z. Guo and G.Q. Xu, Riesz basis and exact controllability of $C_{0}$-groups with one-dimensional input operators. Syst. Control Lett. 52 (2004) 221-232. 
[18] B.Z. Guo and G.Q. Xu, Expansion of solution in terms of generalized eigenfunctions for a hyperbolic system with static boundary condition. J. Funct. Anal. 231 (2006) 245-268.

[19] B.Ya. Levin, On bases of exponential functions in L2. Zapiski Math. Otd. Phys. Math. Facul. Khark. Univ. 27 (1961) 39-48 (in Russian).

[20] K.S. Liu and Z. Liu, Exponential decay of energy of vibrating strings with local viscoelasticity. Z. Angew. Math. Phys. 53 (2002) 265-280.

[21] K.S. Liu and B. Rao, Exponential stability for the wave equations with local Kelvin-Voigt damping. Z. Angew. Math. Phys. 57 (2006) 419-432.

[22] Z.H. Luo, B.Z. Guo and Ö. Morgül, Stability and Stabilization of Linear Infinite Dimensional Systems with Applications. Springer-Verlag, London (1999).

[23] A. Pazy, Semigroups of Linear Operators and Applications to Partial Differential Equations. Springer-Verlag, New York (1983).

[24] R. Rebarber, Exponential stability of coupled beams with dissipative joints: a frequency domain approach. SIAM J. Control Optim. 33 (1995) 1-28.

[25] M. Renardy, On the linear stability of hyperbolic PDEs and viscoelastic flows. Z. Angew. Math. Phys. 45 (1994) 854-865.

[26] A.A. Shkalikov, Boundary problems for ordinary differential equations with parameter in the boundary conditions. J. Soviet Math. 33 (1986) 1311-1342.

[27] J.M. Wang and S.P. Yung, Stability of a nonuniform Rayleigh beam with internal dampings. Syst. Control Lett. 55 (2006) 863-870.

[28] G. Weiss and R.F. Curtain, Exponential stabilization of a Rayleigh beam using colocated control. IEEE Trans. Automatic Control (to appear).

[29] G.Q. Xu and B.Z. Guo, Riesz basis property of evolution equations in Hilbert spaces and application to a coupled string equation. SIAM J. Control Optim. 42 (2003) 966-984.

[30] G.Q. Xu and S.P. Yung, Stabilization of Timoshenko beam by means of pointwise controls. ESAIM: COCV 9 (2003) 579-600.

[31] R.M. Young, An Introduction to Nonharmonic Fourier Series. Academic Press, Inc., London (1980). 